\title{
PARTICLE IMPACT DAMPING IN THE HORIZONTAL PLANE
}

\author{
A Thesis \\ by \\ BRYAN LEE WITT \\ Submitted to the Office of Graduate Studies of \\ Texas A\&M University \\ in partial fulfillment of the requirements for the degree of \\ MASTER OF SCIENCE
}

May 2011

Major Subject: Aerospace Engineering 


\title{
PARTICLE IMPACT DAMPING IN THE HORIZONTAL PLANE
}

\author{
A Thesis \\ by \\ BRYAN LEE WITT
}

\begin{abstract}
Submitted to the Office of Graduate Studies of Texas A\&M University in partial fulfillment of the requirements for the degree of

MASTER OF SCIENCE
\end{abstract}

\begin{abstract}
Approved by:
Chair of Committee, Vikram Kinra

Committee Members, James Boyd Alan Palazzolo

Head of Department, Dimitris Lagoudas
\end{abstract}

May 2011

Major Subject: Aerospace Engineering 


\author{
ABSTRACT \\ Particle Impact Damping in the Horizontal Plane. (May 2011) \\ Bryan Lee Witt, B.S., The University of Texas at Tyler \\ Chair of Advisory Committee: Dr. Vikram Kinra
}

Particle impact damping is measured for a cantilevered beam vibrating freely in the horizontal plane. Several particle configurations are investigated beginning with a single particle and progressing to multiple layers of particles. The effects of clearance between the particles and enclosure, initial displacement of the primary system, repeatability of damping, and damping efficiency per unit mass added are evaluated for each particle configuration. The investigation shows that the particle configuration significantly affects damping. Configurations with the fewest particles per cavity demonstrate higher damping efficiency per unit mass. Generally, for configurations with a definable clearance between the particles and enclosure, damping is shown to be a function of the clearance and initial system displacement. For configurations with multiple layers of particles, for which horizontal clearance between the particles and enclosure has no meaning, a new dimensionless parameter which captures the geometry of the particle arrangement is proposed. 
This thesis is dedicated to my loving parents and incredible wife for their tireless support and encouragement. 


\section{ACKNOWLEDGMENTS}

This work would not have been possible without the constant guidance and assistance of my advising professor, Dr. Vikram Kinra. I thank him wholeheartedly for the many hours of professional and personal time he devoted to helping me in this endeavor. Likewise, I would like to thank the staff of the Texas A\&M Aerospace Engineering Department, particularly Karen Knabe and Miriam Alderete, for their time and help in keeping me on track. Finally, I would like to thank my co-workers, especially Marie Cullen, David Neff, Dwight Doty and Jeff Church, for their support and generous assistance in completing this thesis. 


\section{NOMENCLATURE}

\begin{tabular}{|c|c|}
\hline$a$ & particle acceleration amplitude \\
\hline$A$ & primary mass acceleration amplitude \\
\hline$d$ & clearance between enclosure and particles \\
\hline$D$ & particle diameter \\
\hline EI & beam flexural stiffness \\
\hline$g$ & acceleration due to gravity $\left(9.81 \mathrm{~ms}^{-2}\right)$ \\
\hline$H$ & enclosure fill ratio $\left(h_{p} / h_{e}\right)$ \\
\hline$h_{e}$ & height of enclosure cavity normal to plane of vibration \\
\hline$h_{p}$ & height of particle bed in the direction of gravity \\
\hline$K$ & reduced stiffness of the beam \\
\hline$l_{e}$ & length of enclosure cavity in direction of vibration \\
\hline$L$ & length of the beam \\
\hline$m_{b}$ & mass of the beam \\
\hline$m_{\text {encl }}$ & mass of enclosure \\
\hline$m_{p}$ & mass of the particle(s) \\
\hline$M$ & primary mass (reduced mass of the beam) \\
\hline$n$ & number of layers in a particle bed \\
\hline$N$ & number of particles \\
\hline$r$ & particle radius \\
\hline$R$ & effective coefficient of restitution \\
\hline$t$ & time \\
\hline
\end{tabular}




\begin{tabular}{|c|c|}
\hline$T$ & maximum kinetic energy during a cycle \\
\hline$\Delta T$ & kinetic energy dissipated in one cycle \\
\hline$u$ & particle displacement amplitude \\
\hline$U$ & primary mass displacement amplitude \\
\hline$v$ & particle velocity amplitude \\
\hline$V$ & primary mass velocity amplitude \\
\hline$w_{e}$ & width of the enclosure cavity normal to direction of motion \\
\hline$\alpha$ & dimensionless displacement amplitude, single layer of particles $(U / d)$ \\
\hline$\beta$ & dimensionless displacement amplitude, multiple layers $\left(U /\left(h_{p}{ }^{2} h_{e}{ }^{-1}\right)\right)$ \\
\hline$\Gamma$ & dimensionless acceleration amplitude $\left(U \omega^{2} / g\right)$ \\
\hline$\delta$ & particle diameter to particle bed depth ratio $\left(D / h_{p}\right)$ \\
\hline$\gamma$ & mass per unit length of the beam \\
\hline$\mu$ & mass ratio $\left(m_{p} / M\right)$ \\
\hline$\xi$ & beam mode shape normalization factor $(x / L)$ \\
\hline$\sigma$ & particle diameter population standard deviation \\
\hline$\tau$ & dimensionless time $(\omega t)$ \\
\hline$\varphi_{i}$ & eigenfunction corresponding to $i^{\text {th }}$ flexural mode \\
\hline$\Psi$ & Specific damping capacity \\
\hline$\Psi_{m}$ & Mass normalized specific damping capacity $\left(\Psi(1+\mu)^{2} \mu^{-1}\right)$ \\
\hline
\end{tabular}




\section{TABLE OF CONTENTS}

\section{Page}

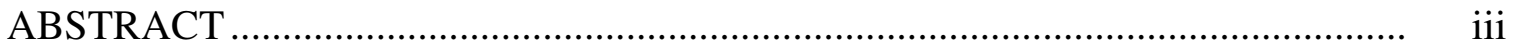

DEDICATION .................................................................................... iv

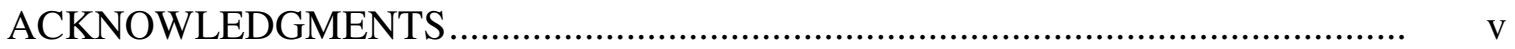

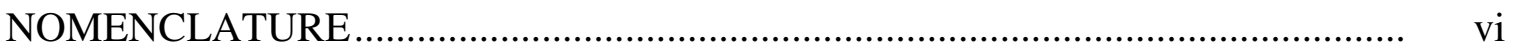

TABLE OF CONTENTS ....................................................................... viii

LIST OF FIGURES ..............................................................................

LIST OF TABLES ..............................................................................

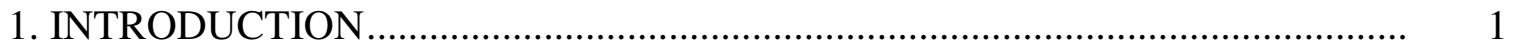

1.1 Particle Impact Damping................................................................ 1

1.2 Overview of Particle Impact Damping Literature .................................. 2

1.3 Objective ...................................................................................... 7

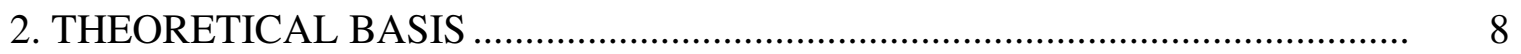

2.1 Vibration of a Cantilevered Beam with an End Mass ............................. 8

2.2 Particle-Enclosure Motion and Specific Damping Capacity..................... 11

2.3 Conditions for Optimal Damping ....................................................... 16

3. EXPERIMENTAL PROCEDURES ….......................................................... 23

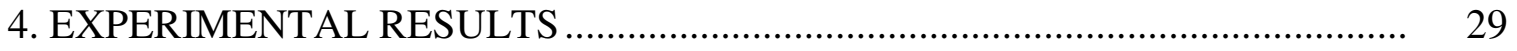

4.1 Configuration 1: Single Cavity Containing a Single Particle .................. 29

4.2 Configuration 2: Three Cavities Each Containing a Single Particle ......... 34

4.3 Configuration 3: Three Cavities Each Containing Two Particles ............. 42

4.4 Configuration 4: Single Horizontal Layer of Particles............................ 44

4.5 Configuration 5: Multiple Layers of Particles.................................... 51

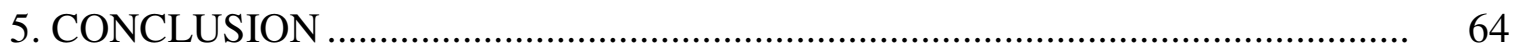

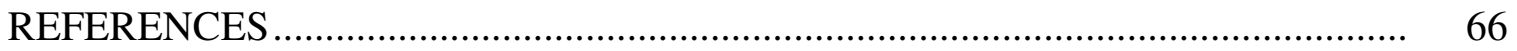


Page

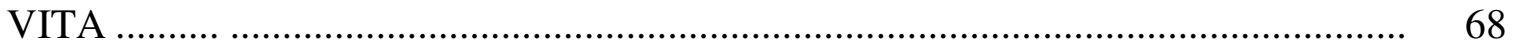




\section{LIST OF FIGURES}

FIGURE $\quad$ Page

2.1 Schematic of beam and enclosure as viewed from above ........................ 9

2.2 Typical damped velocity waveform ................................................ 15

2.3 Simulated motion of an enclosure and point mass particle for optimal damping....

2.4 Simulated motion of an enclosure and point mass particle for minimum damping

2.5 Simulated motion of an enclosure and point mass particle for immediate recapture

2.6 Simulated motion of an enclosure and point mass particle for no impact during a full cycle

3.1 Schematic of experimental setup.......

3.2 Schematic of enclosure dimensions ................................................... 25

4.1 Configuration 1: single cavity containing a single particle.................... 30

4.2 Resin insert dimensions.................................................................... 31

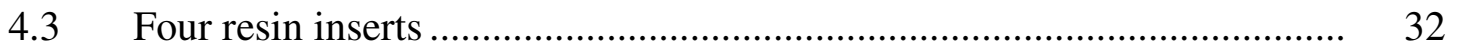

4.4 Mass normalized damping versus displacement amplitude for a single particle; effects of varying $U_{o}$ at four different clearances

4.5 Damping versus displacement amplitude for a single particle; effects of varying clearance $d$.

4.6 Mass normalized damping versus $\alpha$ for a single particle; effects of varying clearance $d$

4.7 Configuration 2: three separate cavities each containing a single particle

4.8 Damping versus $\alpha$ for three cavities each with a single particle; effects of varying clearance $d$ 
4.9 Comparison of damping for two different particle configurations near their peak damping point.....

4.10 Fundamental mode shape and placement of particles along the beam for Configuration 2 .....

4.11 Configuration 3: three separate cavities each containing two particles

4.12 $\Psi_{m}$ versus $\alpha$ for three cavities each containing two particles; effects of varying clearance $d$.

4.13 Comparison of $\Psi_{m}$ for three different particle configurations near their peak damping points

4.14 Configuration 4: single horizontal layer with one row of particles

4.15 Configuration 4: single horizontal layer with four rows of particles

4.16 Single horizontal layer of particles, effects of varying the number of particle rows.

4.17 Configuration 5: multiple layers of particles.

4.18 Particle arrangement assumed for calculating the number of layers $n$ for dampers configured with multiple layers of particles

4.19 Damping versus $\Gamma$ for multiple layers of particles; effects of changing $h_{p}$ by adjusting $m_{p}$.

4.20 Two enclosures with the same $h_{p}$ but different particle diameters

4.21 Damping versus dimensionless acceleration amplitude $\beta$ for multiple layers of particles; effects of changing $h_{p}$ by adjusting $m_{p}$, constant $l_{e}=34.77 \mathrm{~mm}$ 
FIGURE Page

4.22 Damping versus dimensionless acceleration amplitude $\beta$ for multiple layers of particles; effects of changing $h_{p}$ by adjusting $m_{p}$,

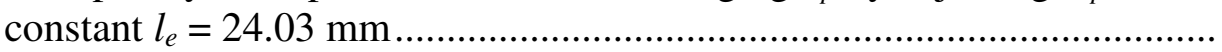

4.23 Damping versus dimensionless acceleration amplitude $\beta$ for multiple layers of particles; effects of changing $h_{p}$ by adjusting $m_{p}$,

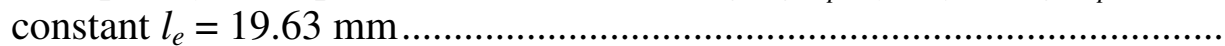




\section{LIST OF TABLES}

TABLE Page

4.2 Test matrix for experiments performed with multiple layers of particles in a single cavity ................................................................ 53 


\section{INTRODUCTION}

\subsection{Particle Impact Damping}

The presence of unwanted vibrations in a structure must often be overcome to avoid damage and eventual failure due to high cycle fatigue. The reduction of these vibrations is achieved by converting the mechanical energy of the structure into thermal and acoustic energy. Damping of structural vibrations can be realized through either active or passive means, the latter being the most common. Many methods of passive damping have been introduced including but not limited to viscoelastic materials, viscous fluids, magnetics and piezoelectrics. The use of viscoelastic materials is one of the most prevalent means of passively damping structural systems. Viscoelastic damping is achieved by converting the vibrating structure's stored elastic strain energy into heat. However, the damping properties of viscoelastic materials are strongly dependent on several parameters including strain rate, aging and temperature. At extremely low and high temperatures viscoelastic material properties are characterized by glassy and flow regions, respectively. In these regimes the damping properties are distinctly diminished. The dependence on the external operating conditions can eliminate viscoelastic materials as a viable damping solution in certain applications, particularly where ambient operating temperatures are more severe, such as in cryogenic environments.

Particle impact damping (PID) is an alternative passive damping method in which a

This thesis follows the style of the Journal of Sound and Vibration. 
particle filled enclosure is attached to a structure undergoing oscillatory vibration. Although the concept of PID is relatively simple there are many damping mechanisms which contribute to the dissipation of energy, resulting in highly nonlinear behavior. As the primary system vibrates, particles within the enclosure undergo inelastic collisions among themselves and the container walls, dissipating the kinetic energy of the structure as heat. Unlike its viscoelastic counterpart PID is essentially independent of the ambient environment allowing it to be used in extreme temperature ranges. Particle impact damping is also less prone to damping property degradation due to age, high cycle fatigue and other permanent time effects. Other attractive attributes of PID include its minimal effects on the strength and stiffness of the structure as well as the ability to generate substantial damping per cycle with a small weight penalty.

\subsection{Overview of Particle Impact Damping Literature}

Impact damping is not a new concept though it has in recent years seen more complex analysis methods and increased applications. Historically the literature has differentiated between impact dampers which contain a single moving mass and particle dampers which contain multiple impacting masses. Many analytical and experimental studies have been presented which generally may be categorized by the direction of excitation in either the vertical (direction of gravity) or horizontal (normal to gravity) plane.

Ema and Marui [1] experimentally investigated the free response of a cantilever beam oscillating in the vertical plane with a single mass impact damper. They report high 
levels of structural damping capability with a small weight penalty and show optimum damping is achieved by combination of the mass ratio and clearance. They also report the critical displacement amplitude below which an impact damper in the vertical plane becomes inactive is a function of the beam frequency and the acceleration of gravity $g$. Butt and Akl [2] performed a numerical analysis of an impact damper applied to a cantilever beam undergoing forced steady-state oscillations. The continuous beam was modeled as a multi-degree of freedom (MDOF) system rather than the more common practice of assuming single degree of freedom (SDOF) behavior. Their analysis considered several factors including the placement of the damper along the length of the beam. In their results they discuss three types of particle-enclosure impacts and find the damper performs best when located away from nodes of the dominant mode shape. They also show the impacts of the damper instantaneously change the velocities of all points of the structure at each impact.

Friend and Kinra [3] carried out an experimental study on a cantilever beam vibrating freely in the vertical plane with a particle damper attached to the free end. They also show very high damping achieved with a small weight penalty. They investigate the effect of particle clearance and displacement amplitude on the specific damping capacity. The fundamental mechanics of impacts with both the floor and ceiling of the enclosure are analytically studied and a simple but effective model is presented. It is also reported that the role of gravity in damping diminishes at large acceleration amplitudes. Their work was continued by Marhadi and Kinra [4] who investigated the 
effects of mass ratio, material type and particle count on damping for a PID attached to the free end of a cantilever beam freely decaying in the vertical plane.

Duncan, Wassgren and Krousgrill [5] carried out an analytical simulation to study the damping performance of a vertical impact damper under steady sinusoidal excitation using a simple SDOF model similar to that of Friend and Kinra [3]. In their analysis they consider forcing frequency and amplitude, mass ratio, structural damping ratio, clearance and coefficient of restitution. They report as clearance increases so does the amplitude at which maximum damping is observed. Others have presented very good models of particle dampers using more computationally advanced methods. Wu, Liao and Wang [6] used multiphase flow theory of gas-particles to model the free decay of a vertically oscillating cantilever beam with a PID at the free end. The effects of particleparticle and particle-enclosure friction and impact effects are captured in their analysis. Their analysis compares with corresponding experimental data very well with the except for dampers with very high packing ratios. Mao et al. [7] utilized the discrete element method (DEM) to simulate particle damping for a cantilever beam vibrating vertically. A comparison of the DEM simulation to the experimental work of Friend and Kinra [3] shows good agreement. Damping is observed to be highly nonlinear and especially dependent on the displacement amplitude, frequency and gravity. Furthermore, the mechanics of energy dissipation were reported to change with the particle packing ratio and vibration frequency. 
Masri [8] developed an exact solution of the general steady-state response of a forced steady-state horizontally excited system with an impact damper. The motion of the system was taken as a piecewise linear process and a stability analysis of the solution was performed. Results of his work indicate that when dampers are in steady-state motion with two impacts per cycle the impacts are typically equispaced in time and produce symmetric motion of the primary system about its equilibrium point. Moore et al. [9] performed a forced response analysis of an impact damper applied to a SDOF oscillator. Simulation results were used to design impact dampers which were applied to an experimental rotor bearing system under cryogenic conditions. McElhaney, Palazzolo and Kascak [10] modeled impact dampers applied to MDOF structures with three-dimensional beam finite elements. Good accuracy and substantial savings in computational time were demonstrated by conducting simulations in the modal subspace rather than full DOF physical coordinate space. A simulation of the rotor bearing system from Moore et al. [9] was performed and showed excellent agreement with the previous experimental results.

Other experimental investigations of impact dampers applied to free horizontal vibrations have been conducted by Yasuda and Toyoda [11] as well as Bapat and Sankar [12]. The former paper investigates mass ratio and coefficient of restitution as well as the effects of using a combined damper comprised of two individual dampers was investigated. The damping performance of the combined damper was found to effectively be the sum of the damping due to the individual units. Bapat and Sankar [12] 
carried out a similar analysis for a single unit impact damper but additionally varied the clearance between the primary and secondary masses.

Saluena et al. [13] investigated the overall dissipative properties of particle dampers through the use of molecular dynamic simulation. By studying the rate of energy dissipation they show the granular system operates in three different damping regimes in the excitation force's amplitude-frequency plane. The granular system exhibits solid, convective and gas like regimes, of which the latter is presented as the most promising for effective particle damping. Saeki $[14,15]$ has also successfully employed DEM to model SDOF systems with both single and multiple particle dampers operating under forced steady-state excitation in the horizontal plane. Good agreement is found between experimental and analytical results for the amplitude reduction at system resonance. In tests with multiple particle dampers only the number of PID enclosures and locations were examined; no variations of the particles within each enclosure were considered.

Papalou and Masri [16] experimentally investigated particle dampers applied to a SDOF structure under horizontal harmonic excitation. The effects of particle size, enclosure dimensions as well as excitation amplitude and frequency were studied. They report high damping with small weight penalties for an optimally designed damper. The use of multiple particles versus a single impacting mass was shown to reduce interface material deterioration, operational noise as well as reduce the sensitivity of the damper to the enclosure dimensions and displacement amplitude. However the penalty for the 
improved performance is a reduction the damping efficiency per unit mass that can be achieved with a single particle damper. Popplewell and Semercigil [17] examined the performance of a particle filled bean bag impact damper. Their experimental setup simulated a SDOF structure vibrating horizontally due to sinusoidal excitation. One challenge presented is the bean bag does not have a unique clearance as a rigid mass does. Results indicate the bean bag provides very good resonant displacement attenuation. Cempel and Lotz [18] performed a similar experimental investigation in which they test several different shot packing methods such as loose, in a soft plastic bag or "rocking" bag with a weighted base. They found the acceleration levels at which the various shot filled containers became inactive can be quite high. The highest energy dissipation was reported for a tightly packed plastic or mesh bag, similar to that examined by Popplewell and Semercigil [17].

\subsection{Objective}

Particle impact damping is measured for a cantilevered beam vibrating freely in the horizontal plane. It is the objective of this work to experimentally explore the effect of the particle configuration within the enclosure. For each particle configuration the effects of clearance between the particles and enclosure as well as the initial displacement of the primary system are evaluated. Repeatability of damping and effectiveness per unit mass added are considered. 


\section{THEORETICAL BASIS}

\subsection{Vibration of a Cantilevered Beam with an End Mass}

A schematic of the beam and particle enclosure is shown in Fig. 2.1a. As depicted in Fig. 2.1b the PID enclosure is idealized as a point mass $m_{e}$ attached to the free end of a cantilevered Euler-Bernoulli beam of length $L$, i.e., the deflection of the beam is assumed to be due to the bending moment only and the effects of shear deformation and rotary inertia are neglected. The governing fourth order partial differential equation of motion is

$$
\frac{\partial^{2} U}{\partial t^{2}}+\frac{E I}{\gamma} \frac{\partial^{4} U}{\partial x^{4}}=0
$$

where $E I$ is the flexural stiffness of the beam and $\gamma$ is the mass per unit length [19]. The boundary conditions for a fixed-free beam of length $L$ carrying a lumped mass, $m_{e}$, at its free end are

$$
\begin{gathered}
U(0, t)=0 \\
\frac{\partial U(0, t)}{\partial x}=0 \\
E I \frac{\partial^{2} U(L, t)}{\partial x^{2}}=0 \\
E I \frac{\partial^{3} U(L, t)}{\partial x^{3}}=m_{e} \frac{\partial^{2} U(L, t)}{\partial t^{2}}
\end{gathered}
$$

The first two boundary conditions state the displacement and slope at the clamped end of the beam must be zero. The third condition requires the bending moment to be zero at the free end of the beam. The final boundary condition requires the shear force at $x=L$ 

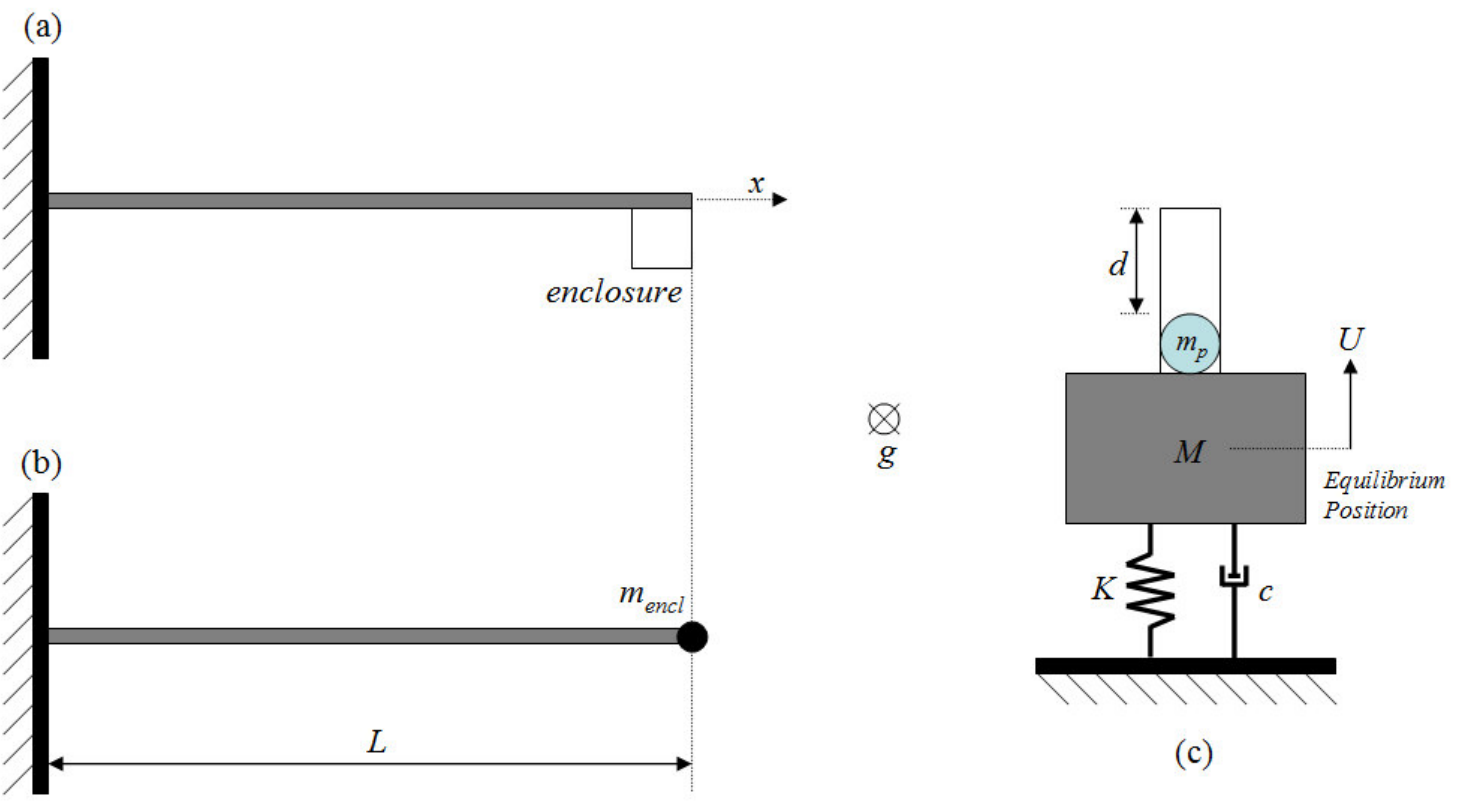

Fig. 2.1. (a) Schematic of beam and enclosure as viewed from above (not to scale). (b) Idealized model of the beam and end mass, $m_{\text {encl }}$. (c) Equivalent single degree of freedom system as viewed from above. 
be equal to the product of the enclosure mass and its acceleration. Assuming the motion of the beam is harmonic and using the method of separation of variables, the displacement solution can be written

$$
U(x, t)=b(x) q(t)=b(x) \cos \left(\omega_{i} t-\phi\right)
$$

for $i=(1,2,3, \ldots, \infty)$. Eq. (2.1) then becomes an eigenvalue problem

$$
\begin{gathered}
\frac{d^{4} b(x)}{d x^{4}}-\lambda_{i}^{4} b(x)=0 \\
\lambda_{i}^{4}=\frac{\omega_{i}^{2} \gamma}{E I}
\end{gathered}
$$

for which the solution is

$$
b(x)=C_{1} \sin \left(\lambda_{i} x\right)+C_{2} \cos \left(\lambda_{i} x\right)+C_{3} \sinh \left(\lambda_{i} x\right)+C_{4} \cosh \left(\lambda_{i} x\right)
$$

where $C_{n}$ are arbitrary constants [20]. The application of the boundary conditions from Eqs. (2.2) thorough (2.5) leads to the familiar eigenvalue solution

$$
\left[\begin{array}{cc}
\left(\sinh \theta_{i}+\sin \theta_{i}\right) & \left(\cosh \theta_{i}+\cos \theta_{i}\right) \\
\Omega_{i}\left(\sinh \theta_{i}-\sin \theta_{i}\right)+\cosh \theta_{i}+\cos \theta_{i} & \Omega_{i}\left(\cosh \theta_{i}-\cos \theta_{i}\right)+\sinh \theta_{i}-\sin \theta_{i}
\end{array}\right]\left[\begin{array}{l}
C_{1} \\
C_{2}
\end{array}\right]=[0]
$$

where $\theta_{i}=\lambda_{i} L$ and $\Omega_{i}=m_{e} \theta_{i} /(\gamma L)$. For nontrivial solutions of $C_{1}$ and $C_{2}$, the determinant of the coefficient matrix must be zero. This produces the frequency equation

$$
\Omega_{i}\left(\sinh \theta_{i} \cos \theta_{i}-\cosh \theta_{i} \sin \theta_{i}\right)+\cosh \theta_{i} \cos \theta_{i}=-1
$$

The roots of Eq. (2.10) can be determined using numerical means such as the NewtonRhapson method. We next define $\xi=x / L$ and the normalized mode shape $\phi_{i}(\xi)$ of the $i^{\text {th }}$ mode is given by [21]

$$
\phi_{i}(\xi)=\sinh \left(\theta_{i} \xi\right)-\sin \left(\theta_{i} \xi\right)-\left(\frac{\sin \theta_{i}+\sinh \theta_{i}}{\cos \theta_{i}+\cosh \theta_{i}}\right)\left(\cosh \left(\theta_{i} \xi\right)-\cos \left(\theta_{i} \xi\right)\right)
$$


Throughout this work, it is assumed the vibratory response of the beam is dominated by its fundamental mode, $\left[\omega_{1}, \phi_{1}\right]$.

The continuous beam is reduced to a single degree of freedom (SDOF) at the location $x$ $=L$ as shown in Fig. 2.1c. The reduced mass of the beam, i.e. the primary mass of the SDOF system, is expressed as

$$
M=m_{e}+\gamma \int_{0}^{L}\left[\phi_{i}\left(x ; m_{e}\right)\right]^{2} d x
$$

where $\gamma$ is the mass per unit length of the beam and $\phi_{i}(x)$ is given in Eq. (2.11). Similarly, the reduced stiffness of the beam at the location $x=L$ is

$$
K=E I \int_{0}^{L}\left[\frac{d^{2} \phi_{i}\left(x ; m_{e}\right)}{d \xi^{2}}\right]^{2} d x
$$

The undamped natural frequency of the equivalent SDOF system is given by the well known expression $\omega_{n}=\sqrt{K / M}$. Since the exact beam mode shape is used in Eqs. (2.12) and (2.13) the undamped natural frequency of the equivalent SDOF system is exactly equal to that of the beam. Since the intrinsic material damping of the beam used in this work is very small the damped and undamped natural frequencies may be assumed to be equal, i.e., $\omega_{d} \approx \omega_{n}$.

\subsection{Particle-Enclosure Motion and Specific Damping Capacity}

A schematic of a SDOF system with the primary mass at its equilibrium position is shown in Fig. 2.1c. The following exercise is applicable to any cycle of the beam's 
motion. However, for simplicity we refer here to the first cycle of motion where the initial displacement amplitude $U_{o}$ is known. It is assumed the particle is initially positioned next to the wall of the enclosure nearest the initial deflection, as pictured in Fig. 2.1c. The beam is given some initial horizontal displacement $U_{o}$. Upon its release the motion of the enclosure is described by:

$$
\begin{gathered}
U_{1}=U_{o} \cos (\omega t) \\
U_{2}=U_{o} \cos (\omega t)+d+D \\
V=-U_{o} \omega \sin (\omega t) \\
A=-U_{o} \omega^{2} \cos (\omega t)
\end{gathered}
$$

where $U_{l}$ is the displacement of the enclosure wall at which the particles are initially located and $U_{2}$ is the displacement of the enclosure wall opposite of $U_{l}$. The clearance between the particles and the enclosure wall in the direction of vibration is $d$ and the particle diameter is represented by $D$. Initially, the particle will move with the enclosure

$$
\left\{\begin{array}{l}
u=U_{1}+D=U_{o} \cos (\omega t)+D \\
v=V=-U_{o} \omega \sin (\omega t) \\
a=A=-U_{o} \omega^{2} \cos (\omega t)
\end{array}\right\} ; 0 \leq t \leq t_{s}
$$

where $t_{s}$ is the moment the particle separates from the enclosure. Separation will occur when the enclosure begins to decelerate as it passes through its equilibrium point, i.e. when the beam reaches zero deflection:

$$
U_{1}=U_{o} \cos (\omega t)=0
$$

This condition first occurs at the dimensionless time $\tau_{s}=\omega t_{s}=\pi / 2$. Neglecting rolling friction between the particle and enclosure floor the particle will travel at a constant 
velocity until it impacts the enclosure wall opposite its initial location, $u=U_{2}$.

$$
\left\{\begin{array}{l}
u=v\left(t-t_{s}\right)+D \\
v=-U_{o} \omega \\
a=0
\end{array}\right\} ; t_{s}<t<t_{\text {impact }}
$$

Due to kinetic energy dissipation, both the enclosure and particle will have new velocities following an impact. The magnitude of the energy dissipated is heavily influenced by the relative velocity between the enclosure and particle at the moment of impact as well as an effective coefficient of restitution.

In this work the effects of all mechanisms of energy dissipation will be collectively dealt with by establishing an effective coefficient of restitution, $R$ [3]. Let $V$ and $V^{+}$be the velocity of the enclosure before and after an impact respectively. Similarly, let $m_{p}$ be the mass of the particle in the enclosure and $v$ and $v^{+}$be its velocity before and after an impact. Considering a collinear impact between $M$ and $m_{p}$ the effective coefficient of restitution is defined as

$$
R=-\frac{\left(v^{+}-V^{+}\right)}{(v-V)}, \quad 0 \leq R \leq 1
$$

Applying the conservation of linear momentum, the velocities following an impact are

$$
\begin{gathered}
V^{+}=\frac{V(1-\mu R)+v \mu(1+R)}{(1+\mu)} \\
v^{+}=\frac{V(1+R)+v(\mu-R)}{(1+\mu)}
\end{gathered}
$$

where $\mu=m_{p} / M$ is the mass ratio. The amount of kinetic energy dissipated during an 
impact between a particle and the enclosure can be determined using the conservation of energy.

$$
\Delta T=\frac{1}{2}\left(1-R^{2}\right) \frac{m_{p}}{1+\mu}(v-V)^{2}
$$

It can be seen from the expression above that the greatest amount of kinetic energy will be dissipated during a cycle when the relative velocity between the particle and enclosure is the greatest, reminiscent of a head-on collision. This will be investigated further in following sections. In PID the damping arises from a conversion of kinetic energy into heat, thus the specific damping capacity per cycle is defined as [3]

$$
\Psi=\frac{\Delta T}{T}
$$

where $\Delta T$ is the kinetic energy converted into heat during one cycle and $T$ is the maximum kinetic energy during the cycle. By defining a cycle as the interval between two successive maxima of the primary mass velocity waveform, $V_{i}$ and $V_{i+1}$, the kinetic energy $T$ is maximum at the beginning of a cycle (see Fig. 2.2). Thus for the $i^{\text {th }}$ cycle

$$
T_{i}=\frac{1}{2} M V_{i}^{2}
$$

The energy dissipated during a cycle is then expressed as

$$
\Delta T_{i}=T_{i}-T_{i+1}=\frac{1}{2} M\left(V_{i}^{2}-V_{i+1}^{2}\right)
$$

Substituting Eqs. (2.26) and (2.27) into Eq. (2.25) yields an expression for the specific damping capacity during a cycle in terms of the velocity of the primary mass:

$$
\Psi_{i}=\frac{V_{i}^{2}-V_{i+1}^{2}}{V_{i}^{2}}
$$




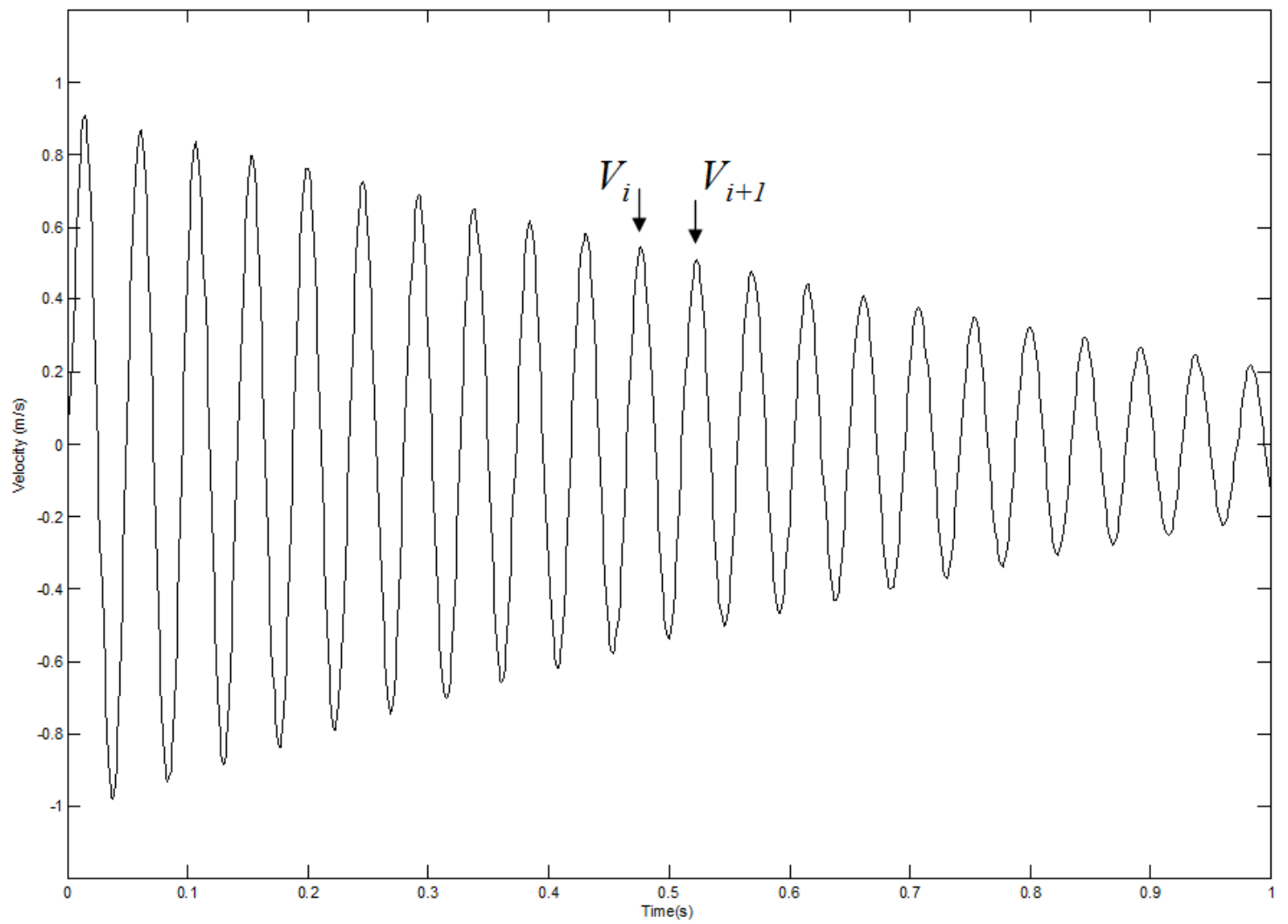

Fig. 2.2. Typical damped velocity waveform. 
Furthermore, as defined by Friend and Kinra [3] the mass normalized specific damping capacity is

$$
\Psi_{m}=\Psi \frac{\left(1+\mu^{2}\right)}{\mu}
$$

which is used to compare experimental results with dissimilar masses.

\subsection{Conditions for Optimal Damping}

Consider a PID system with a given mass ratio $\mu$ vibrating in the direction of gravity. Friend and Kinra [3] have shown the moment of the first impact between the particle and enclosure ceiling is solely a function of $\alpha=U / d$ provided gravity may be neglected (e.g., the decrease in the particle velocity is negligible in comparison to its initial launch velocity). For a system vibrating normal to the direction of gravity with negligible friction between the particle and enclosure we expect the same observation to be valid. To that end, we investigate in more detail the relationship between $\alpha$ and the conditions under which horizontal plane impacts occur.

Eq. (2.24) indicates the maximum kinetic energy dissipated per cycle occurs when the particle and enclosure collide at the moment their relative velocity is greatest. Upon separating from the enclosure the particle velocity is constant and thus at its maximum. The beam will have a maximum velocity in the opposite direction of the particles as its displacement returns back to zero which occurs at dimensionless time $\tau_{\max K E}=\omega t_{\max K E}=$ $3 \pi / 2$ (see Fig. 2.3). Thus for an optimal impact (head-on collision), the particle must 

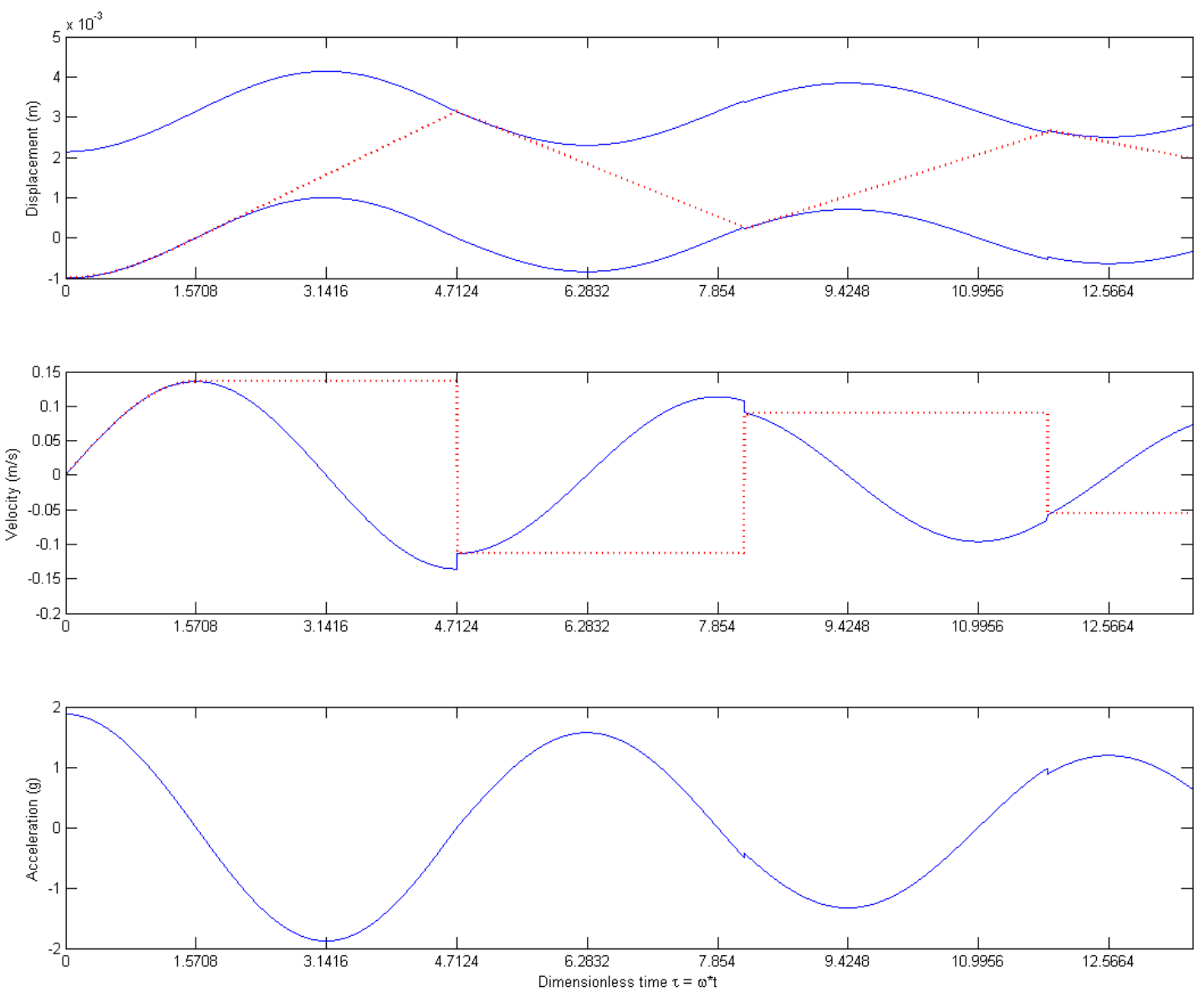

Fig. 2.3. Simulated motion of an enclosure (solid blue) and point mass particle (dashed red) for optimal damping. $U=1 \mathrm{~mm}, d=3.14 \mathrm{~mm}$. 
meet the far wall of the enclosure at time $t_{\max E}$. From Eqs. (2.15) and (2.20) we have

$$
-U_{o} \omega\left(t_{\max K E}-t_{s}\right)=d
$$

Recalling $\alpha=U / d$, Eq. (2.30) leads to the condition for maximum kinetic energy dissipation during an impact as

$$
\alpha=\frac{1}{\pi}
$$

Fig. 2.3 shows a representative system in a cycle where $\alpha=1 / \pi,(U=1 \mathrm{~mm}$ and $d=3.14$ $\mathrm{mm}$ ). Note the first impact occurs at the moment when the relative velocity between the particle and enclosure is the greatest.

Next, we explore the conditions for minimum energy dissipation. Here the velocity of the particle and enclosure should be identical upon impact creating a gentle "catch" effect rather than a collision. With reference to Eqs. (2.18) and (2.20) this happens when the dimensionless time $\tau_{\min K E}=\omega t_{\min K E}=5 \pi / 2$. At this moment the beam is crossing its equilibrium position for the second time just as the particle arrives at the far wall. Substituting $t_{\min K E}$ into Eq. (2.30), the condition for minimum kinetic energy dissipation during an impact is found to be

$$
\alpha=\frac{1}{2 \pi}
$$

This is illustrated in Fig. 2.4. Note that the impact occurs at the moment when the relative velocity between the particle and enclosure is zero.

Eq. (2.31) is a design criterion for maximum damping; in the following it shall be 

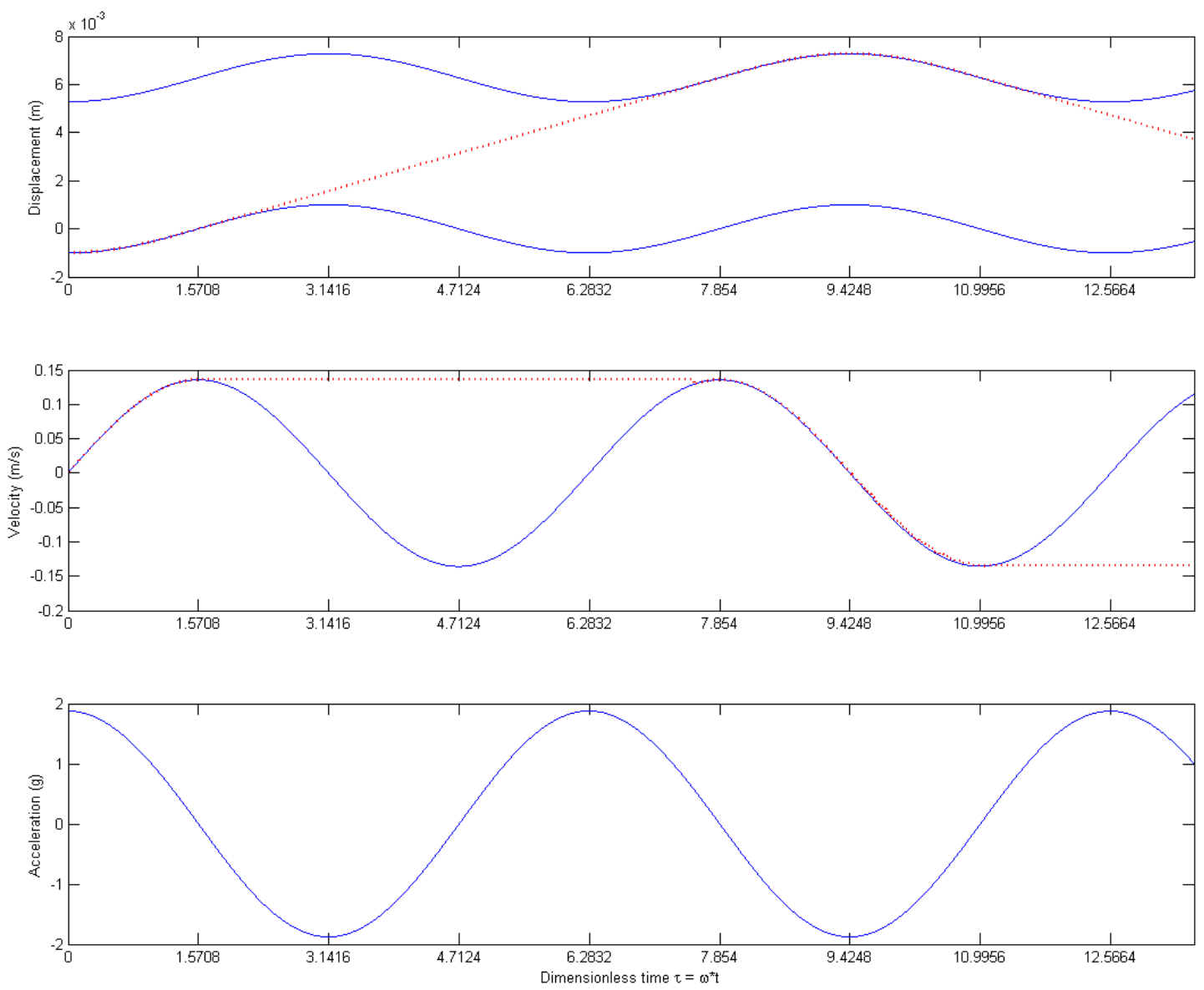

Fig. 2.4. Simulated motion of an enclosure (solid blue) and point mass particle (dashed red) for minimum damping. $U=1 \mathrm{~mm}, d=6.28 \mathrm{~mm}$. 
referred to as the $\alpha$-criterion. We now explore the two other extremes, namely, when $\mathrm{U}$ is very large or very small compared to d. For $\alpha>>1 / \pi$ (i.e. $d<<U$ ), the particle leaves the enclosure and is almost immediately recaptured by the far enclosure wall with a very small velocity difference between the two. A graphical representation of this is shown in Fig. 2.5. At the other extreme, when $\alpha<<1 / \pi$ (i.e. $d>>U$ ), the particle does not impact the far wall during an entire cycle. A graphical representation of this is shown in Fig. 2.6 .

The above analysis specifically pertains to a damper with a single particle. In practical applications many PID systems are built using some configuration of several particles. It is well known that a system of particles does not behave like a single particle under most operating conditions $[7,14,18]$ and, therefore, its behavior will not be accurately described by the $\alpha$-criterion. In the following we report the experimental results obtained with single- and multi-particle systems and interpret them in the context of the single-particle analysis presented above. 

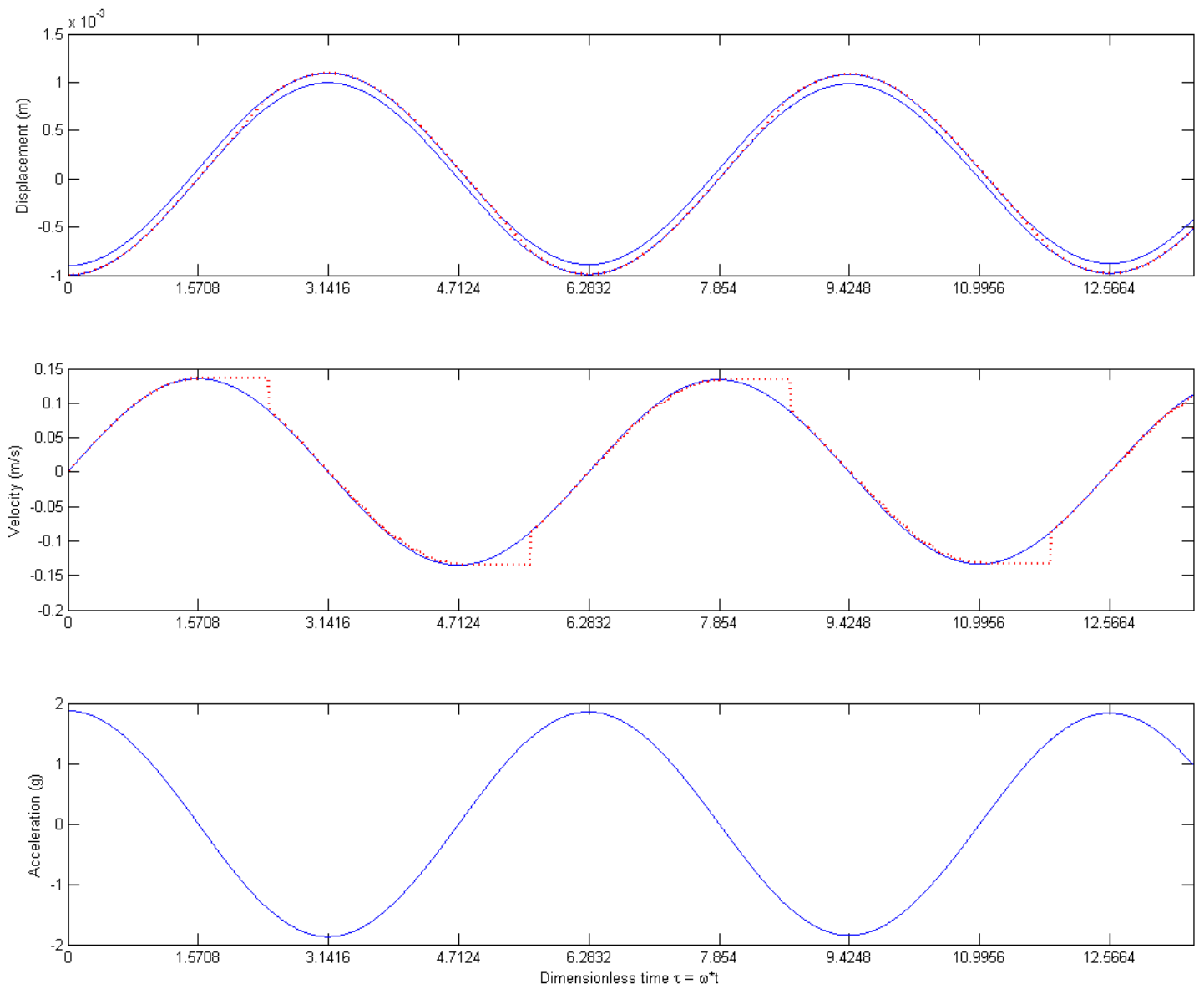

Fig. 2.5. Simulated motion of an enclosure (solid blue) and point mass particle (dashed red) for immediate recapture. Clearance $d=U / 10$. 

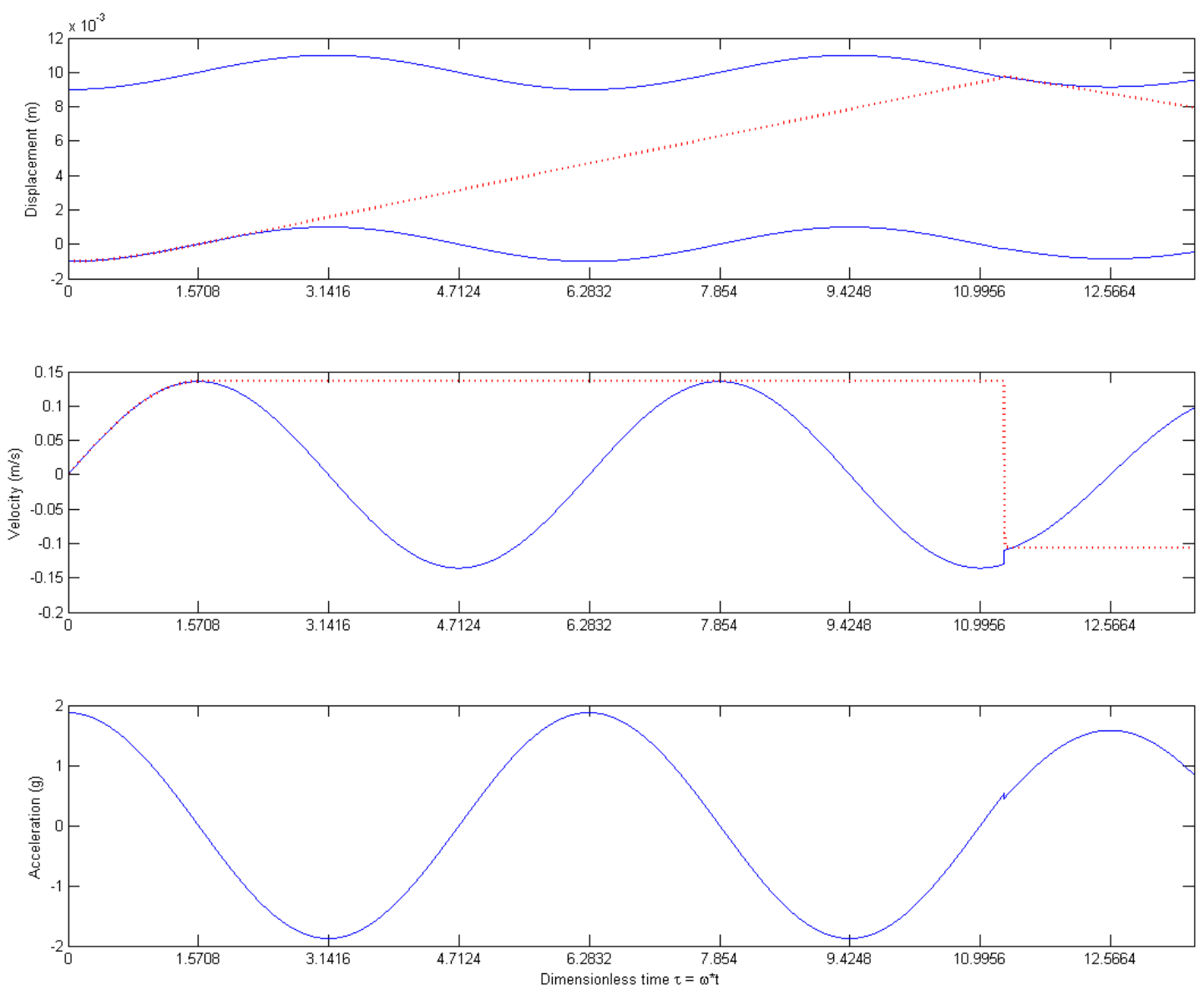

Fig. 2.6. Simulated motion of an enclosure (solid blue) and point mass particle (dashed red) for no impact during a full cycle. Clearance $d=10 U$. 


\section{EXPERIMENTAL PROCEDURES}

A schematic of the experimental setup is shown in Fig. 3.1. The cantilevered beam is made of 4140 steel (Young's modulus $E=207 \mathrm{GPa}$, mass density $\rho=7.84 \times 10^{3} \mathrm{~kg} / \mathrm{m}^{3}$ ). The beam has a width of $19.16 \mathrm{~mm}$, a thickness of $3.19 \mathrm{~mm}$ and length of $244.00 \mathrm{~mm}$. An enclosure is constructed such that the length of the cavity in the direction of motion, $l_{e}$, and the width of the cavity normal to direction of motion, $w_{e}$, are adjustable (see Fig. 3.2a). Enclosure length adjustment is accomplished by attaching the front (impacting) wall to a sliding shaft allowing $l_{e}$ to vary. Interchangeable sidewall inserts are fabricated with specific dimensions that are placed between the front and rear impacting walls. The sidewall inserts are attached to a removable sliding shaft allowing $w_{e}$ to vary as shown in Fig. 3.2b. Set screws are fitted to the shafts to hold them in place during testing. Impacting walls are constructed of aluminum while the remainder of the structure is made of clear polycarbonate allowing visibility of the particles during testing. The maximum dimensions of the cavity are $l_{e}=35.15 \mathrm{~mm}, w_{e}=39.80 \mathrm{~mm}$ and height $h_{e}=$ $35.31 \mathrm{~mm}$. The mass of the enclosure is $m_{e}=102.25$ grams with no sidewall inserts. The beam with the empty enclosure attached at its free end has a nominal fundamental natural frequency of $20 \mathrm{~Hz}$.

The PID enclosure is attached to the free end of the cantilevered beam. The fixed end of the beam is securely clamped to a rigid base. The free end of the beam is then given an initial horizontal displacement $U_{o}$ and held by an electromagnetic coil. The magnitude of the initial displacement is adjusted by moving the position of the coil. At time $t=0$ 


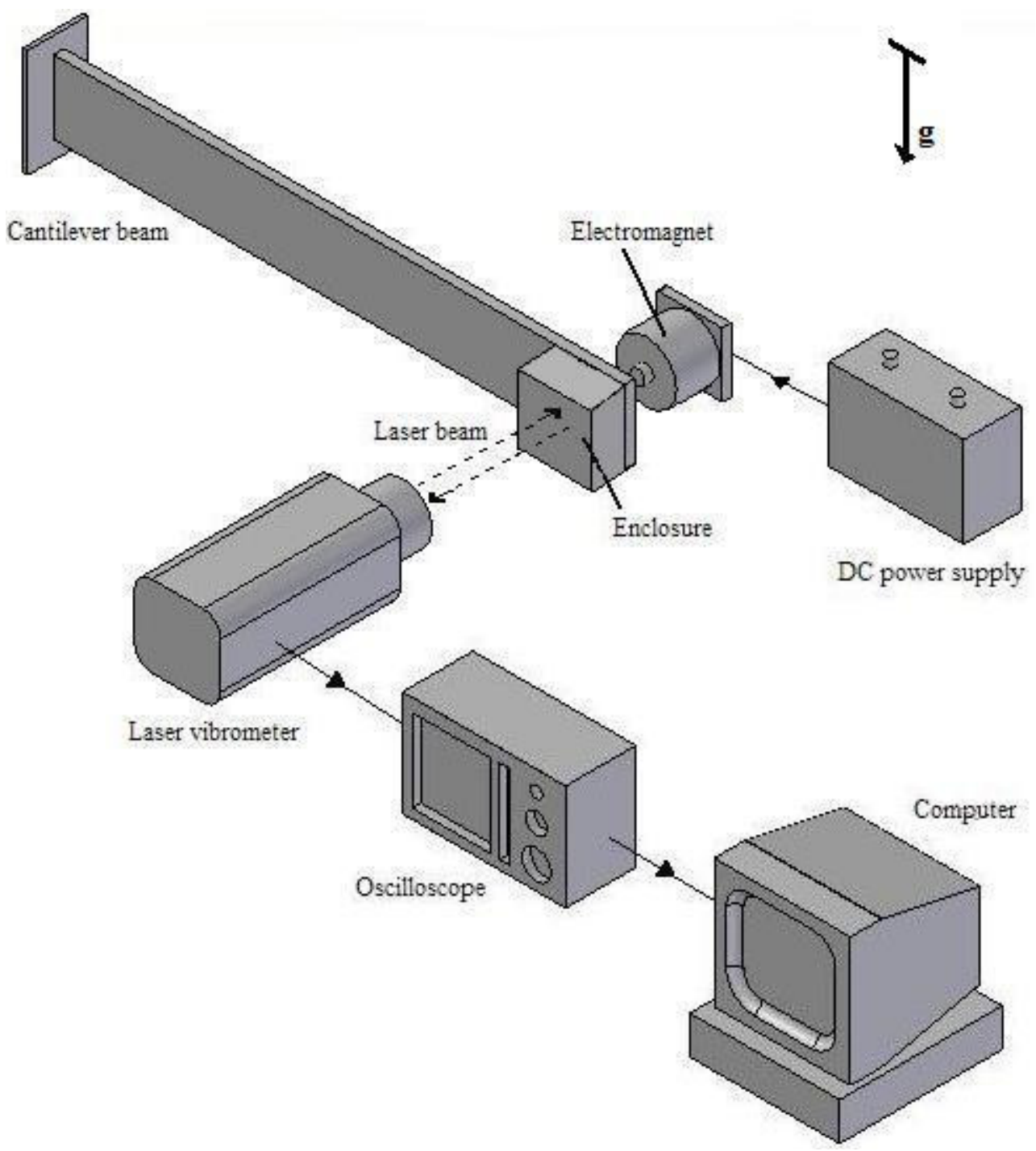

Fig. 3.1. Schematic of experimental setup (not to scale). 

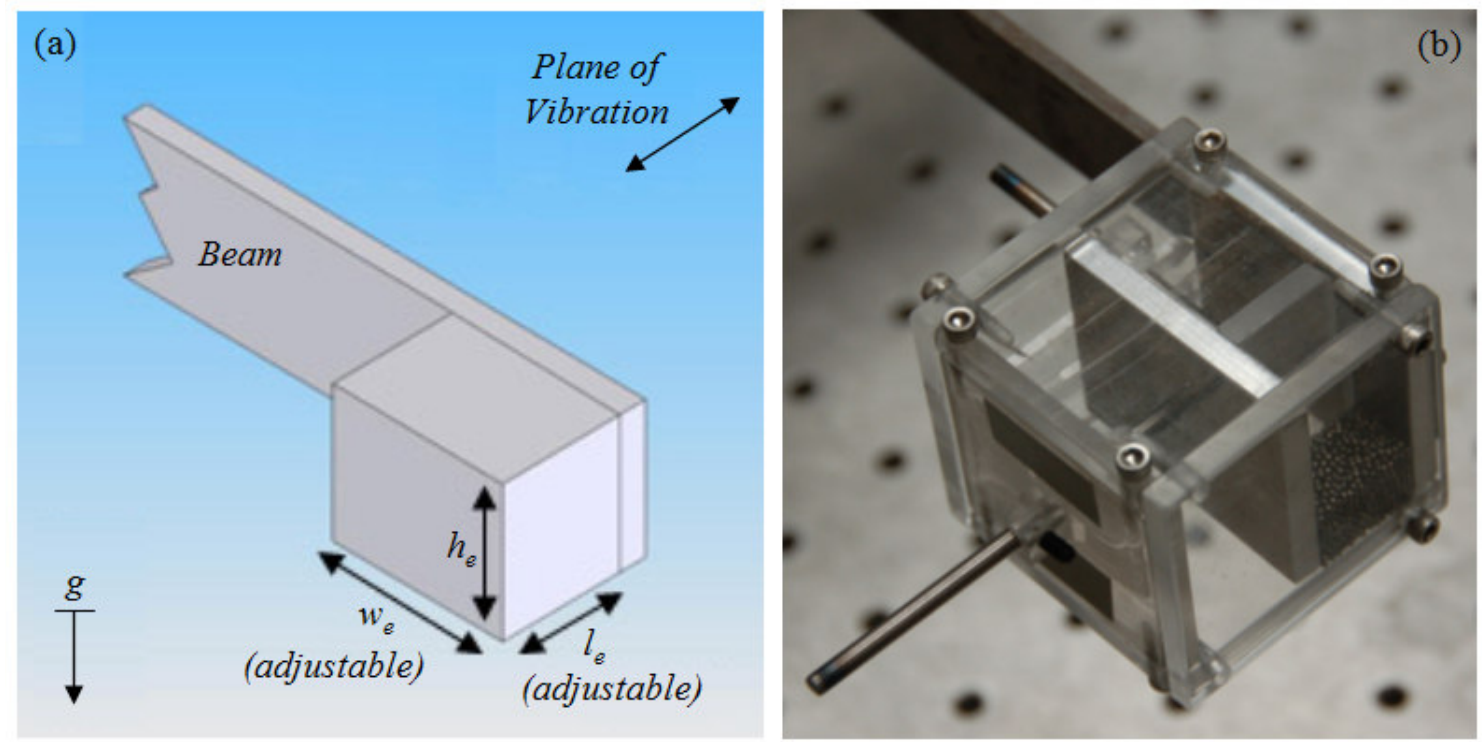

Fig. 3.2. (a) Schematic of enclosure dimensions. (b) Fabricated enclosure with adjustable length and width. 
the power to the electromagnet is terminated and the beam is allowed to decay freely. The resulting velocity waveform of the vibrating beam is captured by a Polytec OFV 302 laser vibrometer sensor head and a Polytec OFV 3000 vibrometer controller positioned in front of the enclosure. A small piece of reflector tape (visible in Fig. 3.2b) is placed at the top-center of the enclosure facing the vibrometer in order to reflect the laser beam back to the sensor head. The Polytec OFV 3000 is capable of measuring the velocity of the enclosure with a resolution of $1.0 \mu \mathrm{m} / \mathrm{s}$.

Data acquisition is triggered as the beam is released and the waveform is recorded by a Yokogawa DL708 digital processing oscilloscope (DPO). The digitizing rate is set at 1000 samples/sec. For the beam frequency of $20 \mathrm{~Hz}$ used in this study the digital sampling rate translates to 50 data points per cycle. The data is then sent to a $\mathrm{PC}$ for post-processing and analysis.

Each test is repeated a minimum of three times under as close to identical conditions as possible. All raw data is processed individually using both the positive and negative peaks of the collected waveforms to calculate damping for each cycle of beam motion, $\Psi_{i}$, as expressed in Eq. (2.28). By measuring the specific damping capacity of the beam with an empty PID enclosure attached, the intrinsic material damping of the beam is determined to be $\Psi=0.3 \%$. To calculate $\Psi$ specifically due to PID, the material damping of the beam is subtracted from the total damping measured in the presence of particles. The results presented herein utilize a three point moving average. Post 
processing and analysis of data is performed in MATLAB $^{\circledR}$ using the equations established in Section 2.

In total three types of spherical particles are used throughout this work. In tests which require a very small number of particles $(N<6)$ steel particles of diameter $D=9.50 \mathrm{~mm}$ are employed. In tests using an intermediate number of particles $(6<N<36)$ steel particles with diameter $D=4.36 \mathrm{~mm}$ are used. For tests involving a large number of particles $(N>36)$ lead particles with $D=1.27 \mathrm{~mm}$ are used. Small steel particles of similar diameter are observed to develop some degree of magnetism from the electromagnet used to hold the beam resulting in undesirable attraction and repulsion between them. Therefore lead is substituted for tests involving small diameter particles. Marhadi and Kinra [4] have shown experimentally that as the number of particles within a PID cavity increases $(N>50) \Psi$ becomes independent of the particle material. All experiments using lead spheres performed in this study have a minimum particle count of $N \approx 1028$.

The steel $4.36 \mathrm{~mm}$ diameter particles were found to have the largest variability in their diameters. A representative selection of 75 particles has a sample standard deviation of $0.013 \mathrm{~mm}$ corresponding to an overall population standard deviation of $\sigma=0.014 \mathrm{~mm}$. For a $99.7 \%$ confidence interval $( \pm 3 \sigma)$ the particle diameter has a variability of $0.08 \mathrm{~mm}$ or less which is sufficiently small to be neglected for multi-particle systems. 
All tests are performed with the beam vibrating in the horizontal plane. Five different configurations of particles within the enclosure are investigated as detailed in the following section. The intent is to begin with a single particle within a single cavity and progress to a granular bed comprising several particles $(N \approx 4000)$. The damping characteristics of each configuration are investigated for varying clearances and initial displacements. 


\section{EXPERIMENTAL RESULTS}

\subsection{Configuration 1: Single Cavity Containing a Single Particle}

The first configuration is a single particle $\left(D=9.50 \mathrm{~mm}, m_{p}=3.52 \mathrm{~g}\right)$ in a cavity with variable length $l_{e}$ in the direction of vibration, fixed height $h_{e}=9.90 \mathrm{~mm}$, and fixed width $w_{e}=12.19 \mathrm{~mm}$. A representative schematic is shown in Fig. 4.1. The cavity is created by placing one of four specially fabricated resin inserts between the enclosure impacting walls. The dimensions of the inserts are shown in Fig. 4.2 and a photograph of the inserts is provided in Fig. 4.3. The inserts have lengths of [10.72, 14.45, 24.38, 29.24] $\mathrm{mm}$ respectively and corresponding masses of $[0.87,1.21,2.02,2.43]$ grams. The masses of the inserts are individually accounted for in the mass normalization of the results. Each insert fits the width of the enclosure and has a specific length which corresponds to a particular value of $l_{e}$. For the single particle configuration the particle is placed in the center cavity which corresponds to the location along the beam where the laser vibrometer measurements are taken.

The clearance between the particle and the enclosure wall $d$ is varied by changing $l_{e}$, i.e. by using a different channel insert. Four values of clearance $d=[1.22,4.95,14.88$, 19.74] $\mathrm{mm}$ are tested. For each clearance experiments are conducted at four values of initial displacement $U_{o}=[2.0,4.0,6.0,8.0] \mathrm{mm}$. The initial displacement is varied by adjusting the electromagnetic coil position. The results of the experiments conducted at each of the four clearances are shown in Figs. 4.4a through 4.4d, respectively. For each value of $d$ tested it is noted that the damping is independent of the initial displacement 


\section{Top View}

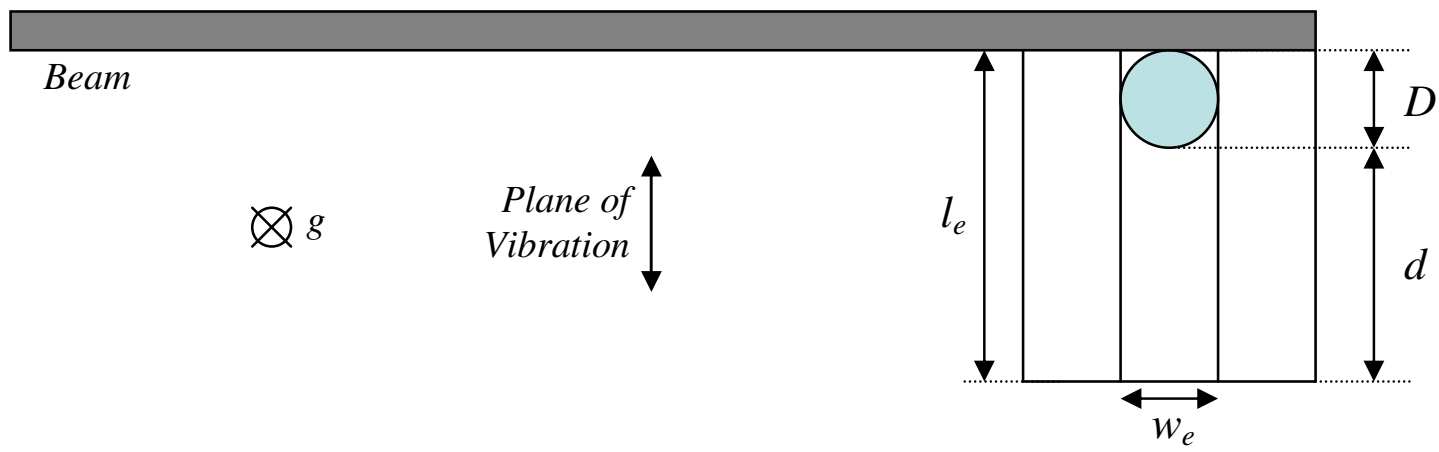

\section{Front View}

\section{Beam}
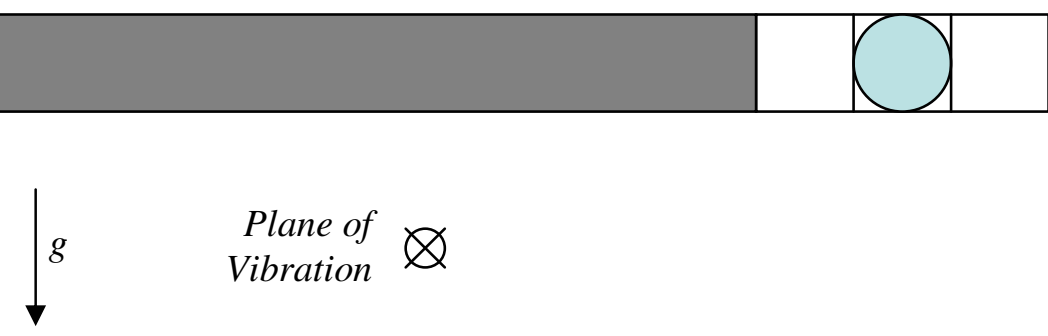

Fig. 4.1. Configuration 1: single cavity containing a single particle (not to scale). $N=$ $1, D=9.50 \mathrm{~mm}, m_{p}=3.52$ grams. 
Top View

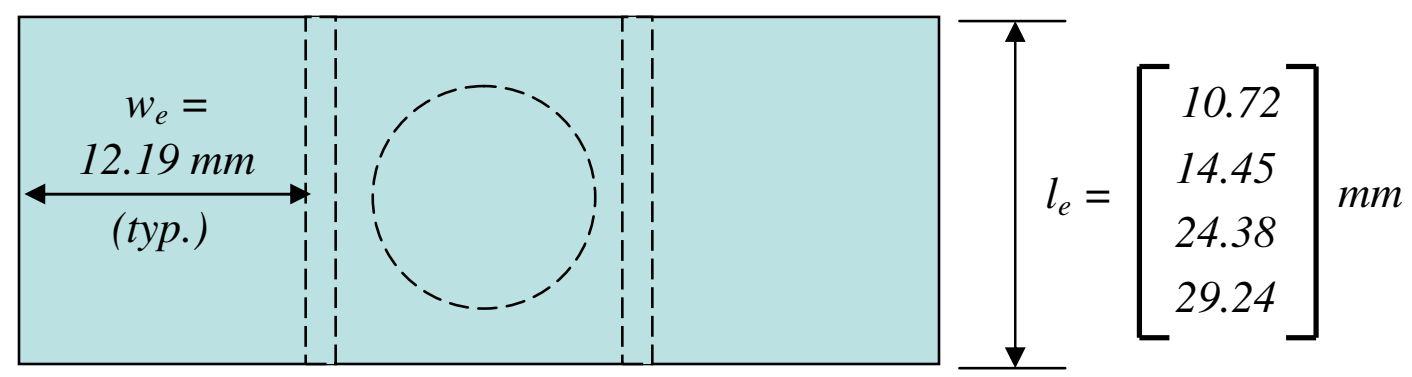

\section{Front View}

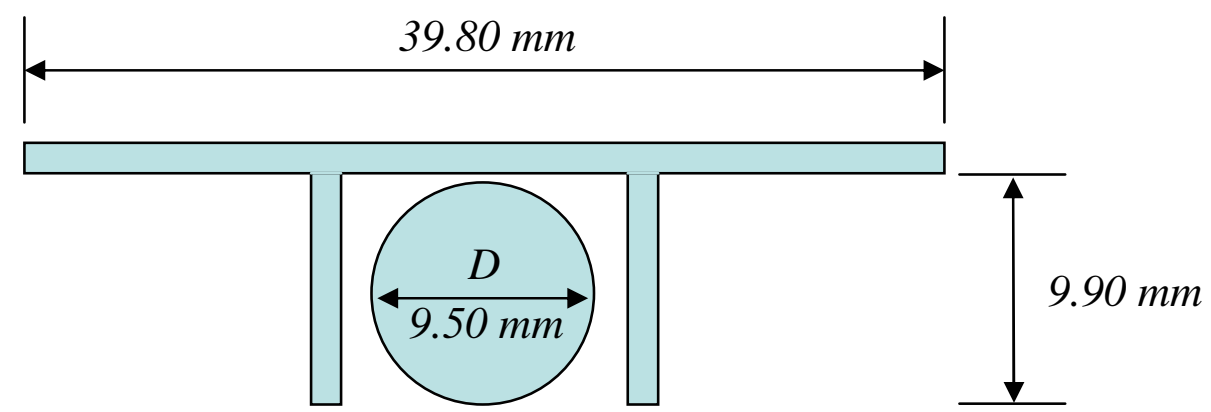

Fig. 4.2. Resin insert dimensions (drawn to scale). 


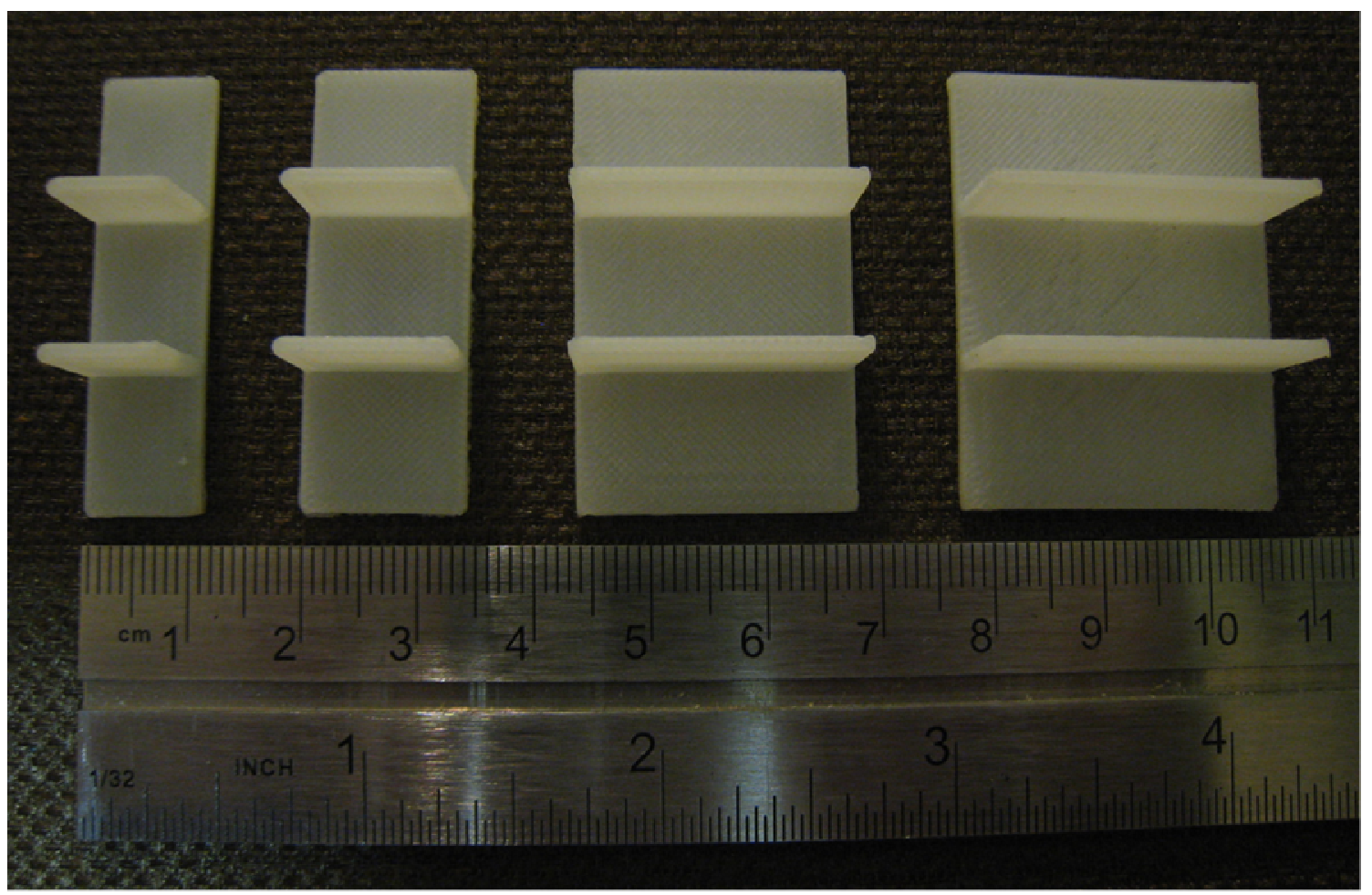

Fig. 4.3. Four resin inserts (pictured lying upside down). 

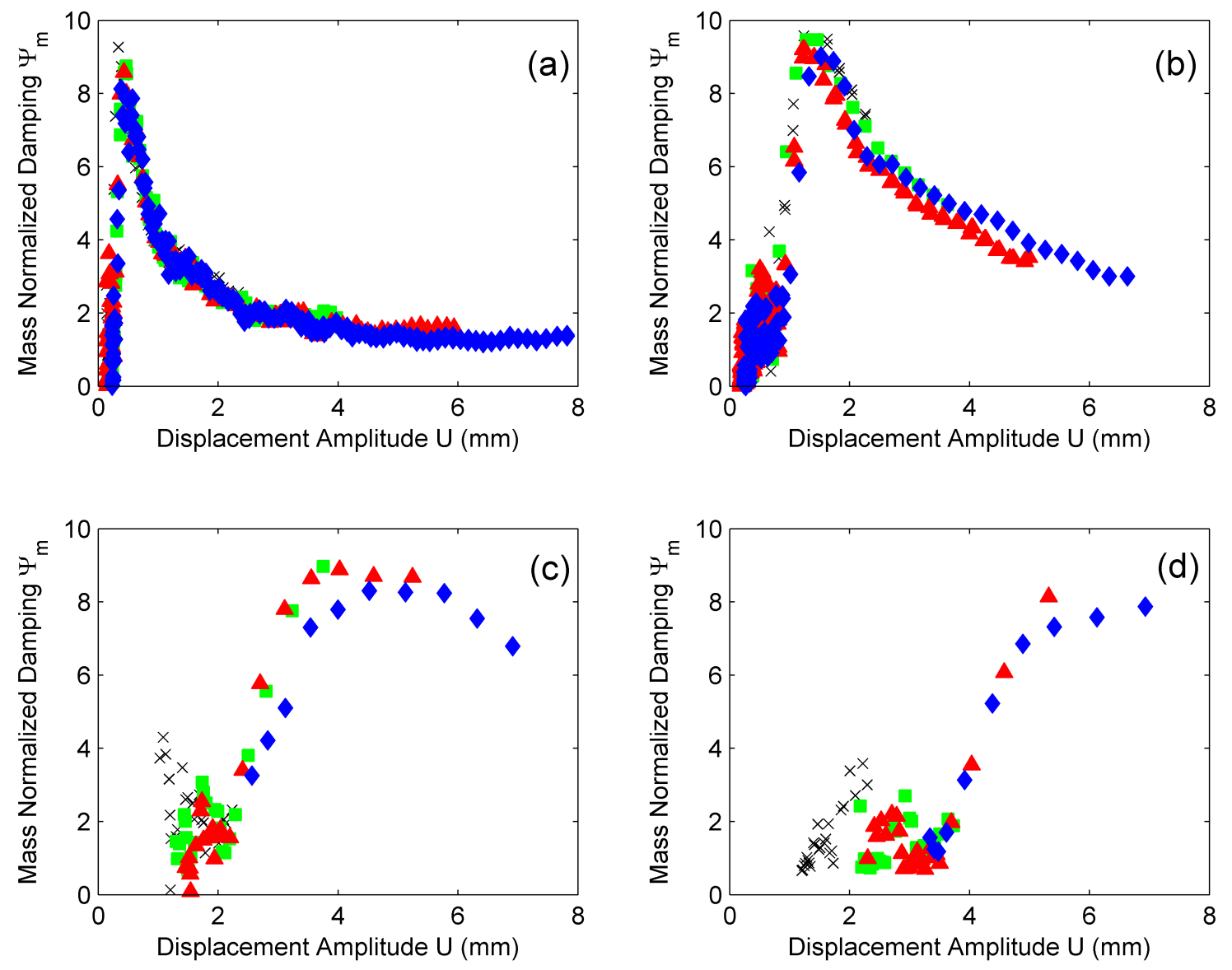

Fig. 4.4. Mass normalized damping versus displacement amplitude for a single particle; effects of varying $U_{o}$ at four different clearances. (a) $d=1.22 \mathrm{~mm}$ (b) $d=4.95 \mathrm{~mm}$ (c) $d=14.44 \mathrm{~mm}$ (d) $d=19.74 \mathrm{~mm}$. In each graph $\diamond U_{o}=2.0 \mathrm{~mm}, \Delta U_{o}=4.0 \mathrm{~mm}, \square U_{o}=$ $6.0 \mathrm{~mm}, \times U_{o}=8.0 \mathrm{~mm}$. 
amplitude $U_{o}$. However, to illustrate that $\Psi_{m}$ is strongly dependent on the clearance $d$ the four graphs in Fig. 4.4 are combined in Fig. 4.5.

From the analytical study of a single particle presented in Section $2.3 \Psi_{m}$ should depend only on the ratio $U / d=\alpha$. Therefore the dimensional data of Fig. 4.5 is plotted as a function of $\alpha$ in Fig. 4.6. It is reassuring to note that the four distinct curves of Fig. 4.5 collapse into a single curve in Fig. 4.6a. The graph in Fig. 4.6b shows in detail the data at lower values of dimensionless displacement amplitude with a solid line denoting the $\alpha$ $=1 / \pi$ (maximum $\Psi$ condition) and a dashed line at $\alpha=1 /(2 \pi)$ (minimum $\Psi$ condition).

The fact that the maximum and minimum damping occur near $1 / \pi$ and $1 /(2 \pi)$ respectively lends credence to the single-particle approximation of damping presented in this work.

The data in Fig. 4.6 has important design implications. For time-harmonic vibrations of a given amplitude $U$ the clearance $d$ is selected to render $\alpha=1 / \pi$ producing maximum damping (tuned damper). Similarly, for transient vibrations the damper is tuned to the peak amplitude.

\subsection{Configuration 2: Three Cavities Each Containing a Single Particle}

The second damper configuration tested comprises three individual chambers each containing a single particle which is identical to that used in the previous configuration: each particle has a diameter of $D=9.50 \mathrm{~mm}$ and an individual mass of $3.52 \mathrm{~g}$ making 


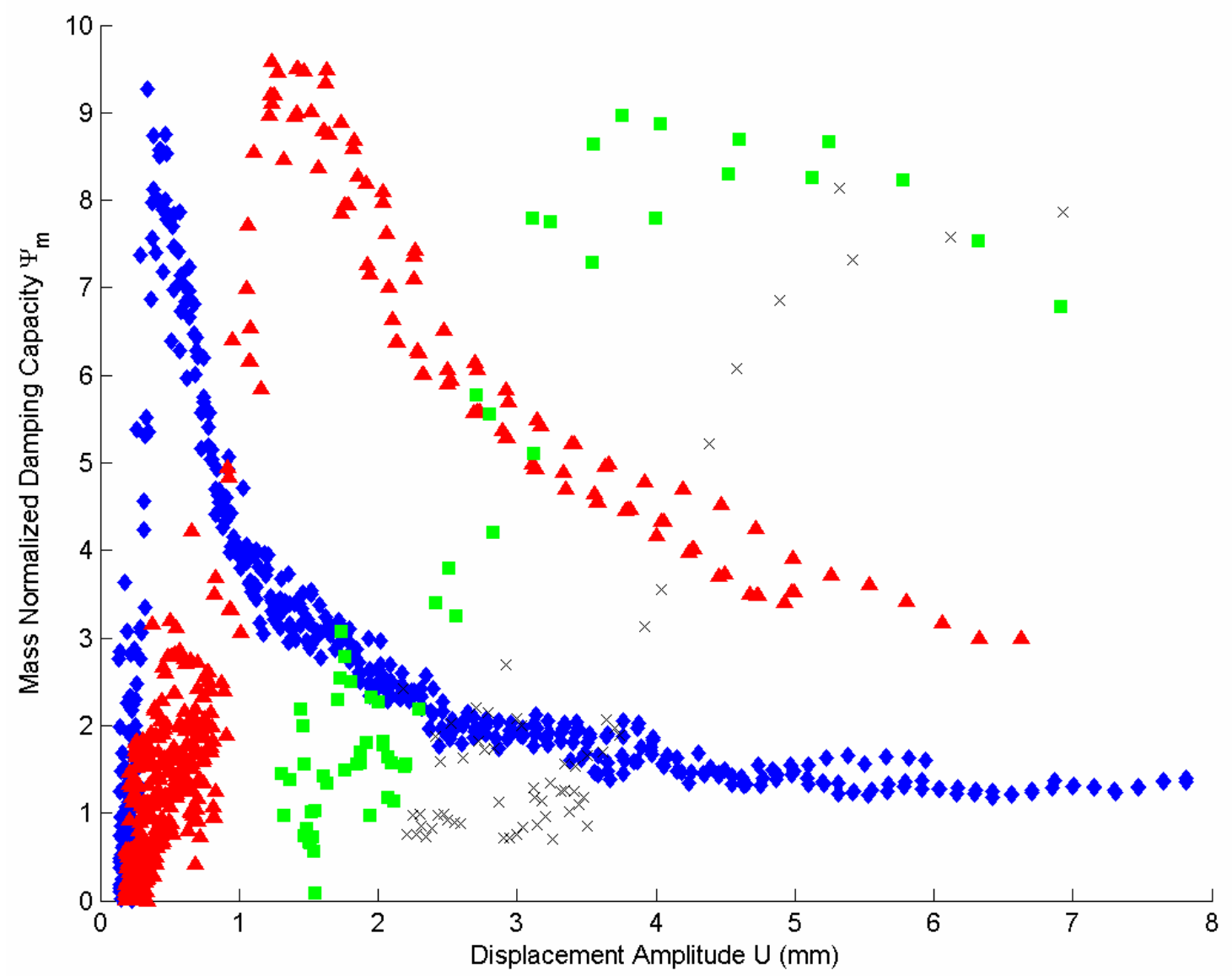

Fig. 4.5. Damping versus displacement amplitude for a single particle; effects of varying clearance $d . d_{1}=1.22 \mathrm{~mm}, \Delta d_{2}=4.95 \mathrm{~mm}, \nabla d_{3}=14.88 \mathrm{~mm}, \times d_{4}=19.74$ $\mathrm{mm}$. All sets contain records with $U_{o}=[2.0,4.0,6.0,8.0] \mathrm{mm}$. 

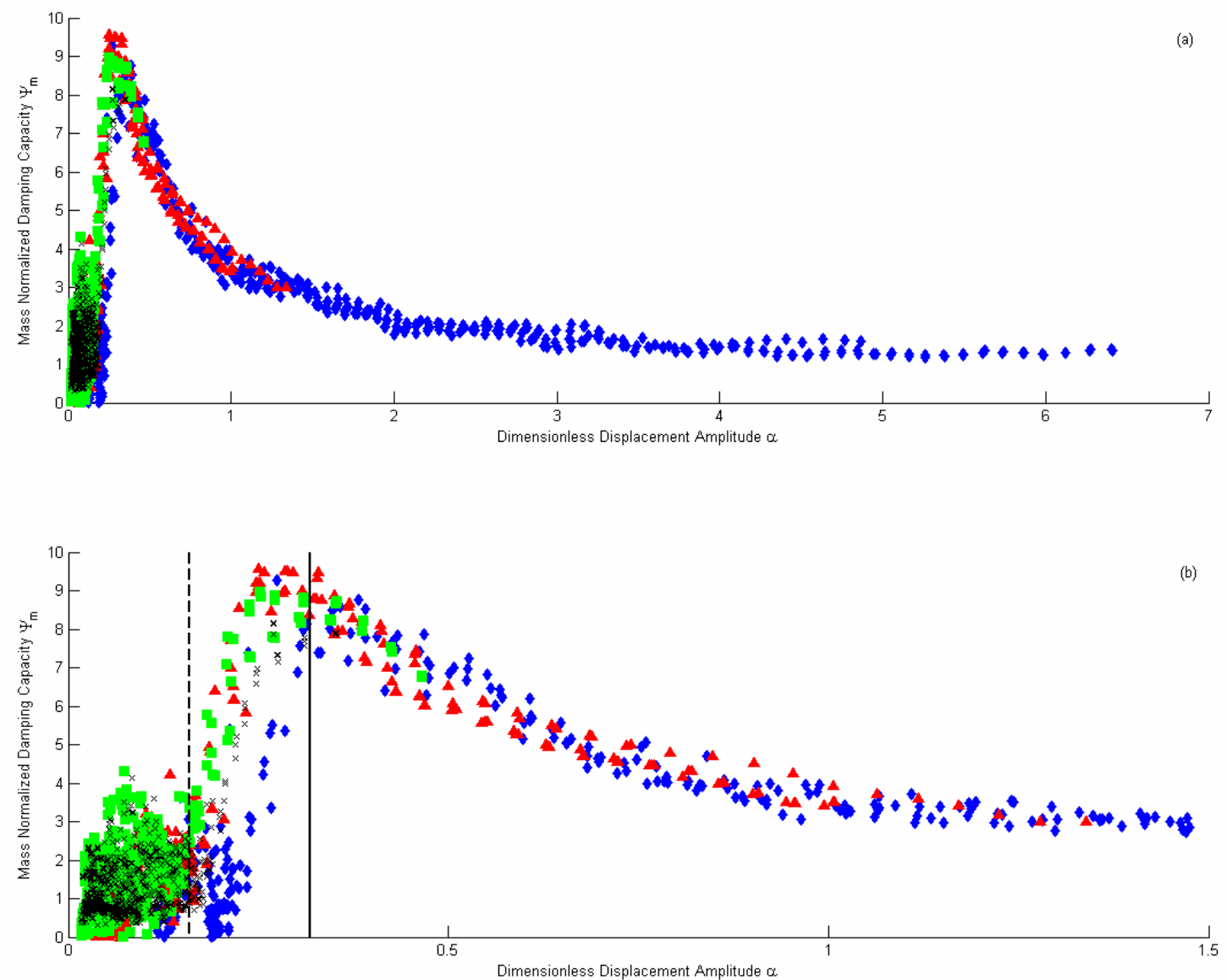

Fig. 4.6. Mass normalized damping versus $\alpha$ for a single particle; effects of varying clearance $d$. (a) Full range of $\alpha$ tested. (b) Detail zoom showing $\alpha=1 / \pi$ (solid line) and $\alpha=1 /(2 \pi)$ (dashed line). $d_{1}=1.22 \mathrm{~mm}, \Delta d_{2}=4.95 \mathrm{~mm}, \quad d_{3}=14.88 \mathrm{~mm}, \times d_{4}=$ $19.74 \mathrm{~mm}$. Each series contains $U_{o}=[2.0,4.0,6.0,8.0] \mathrm{mm}$. 
the total particle mass $m_{p}=10.56 \mathrm{~g}$. All three cavities have the same fixed height $h_{e}=$ $9.90 \mathrm{~mm}$ and fixed width $w_{e}=12.19 \mathrm{~mm}$. As with the previous configuration the enclosure length in the direction of vibration $l_{e}$ is determined by the selection of the resin insert used and is thus identical for all three cavities. A representative schematic is shown in Fig. 4.7. Four values of clearance $d=[1.22,4.95,14.88,19.74] \mathrm{mm}$ are tested with an initial displacement $U_{o}=6.0 \mathrm{~mm}$.

The results of these tests as a function of $\alpha$ are presented in Fig. 4.8. The top graph (a) contains the full range of $\alpha$ over which data was collected. Graph (b) displays a zoom of the above data with a solid line denoting the $\alpha=1 / \pi$ (maximum $\Psi$ condition) and a dashed line at $\alpha=1 /(2 \pi)$ (minimum $\Psi$ condition). Once again it is reassuring to note that the dimensional results for four different values of $d$ collapse into a single curve when plotted as a function of $\alpha$, and that the results are quite reproducible. If the three particles were to move in unison, one would expect the mass-normalized damping for three-particle configuration (Fig. 4.7) to be identical to that due to single-particle configuration (Fig. 4.1); the two are compared in Fig. 4.9. It is noted that the former is significantly smaller (about 25\%) than the latter. It is conjectured that for this particular configuration the three particles do not move in unison.

To support this conjecture we consider the damper dimensions with respect to the fundamental mode shape of the beam as represented in Fig. 4.10. It is clear that the initial displacement and thus launch velocity of each particle is slightly different, $U_{o}^{(1)} \omega$ 


\section{Top View}

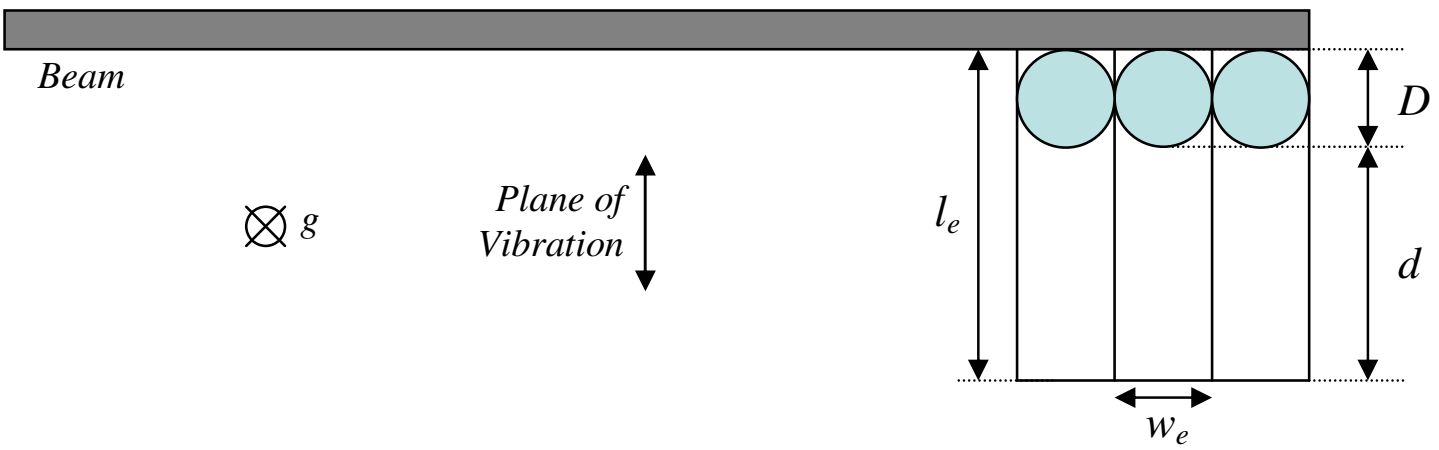

\section{Front View}

\section{Beam}

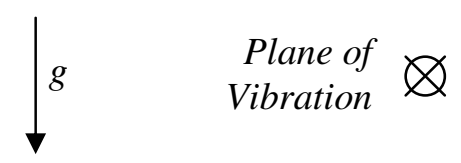

Fig. 4.7. Configuration 2: three separate cavities each containing a single particle (not to scale). $N=3, D=9.50 \mathrm{~mm}, m_{p}=10.56$ grams. 

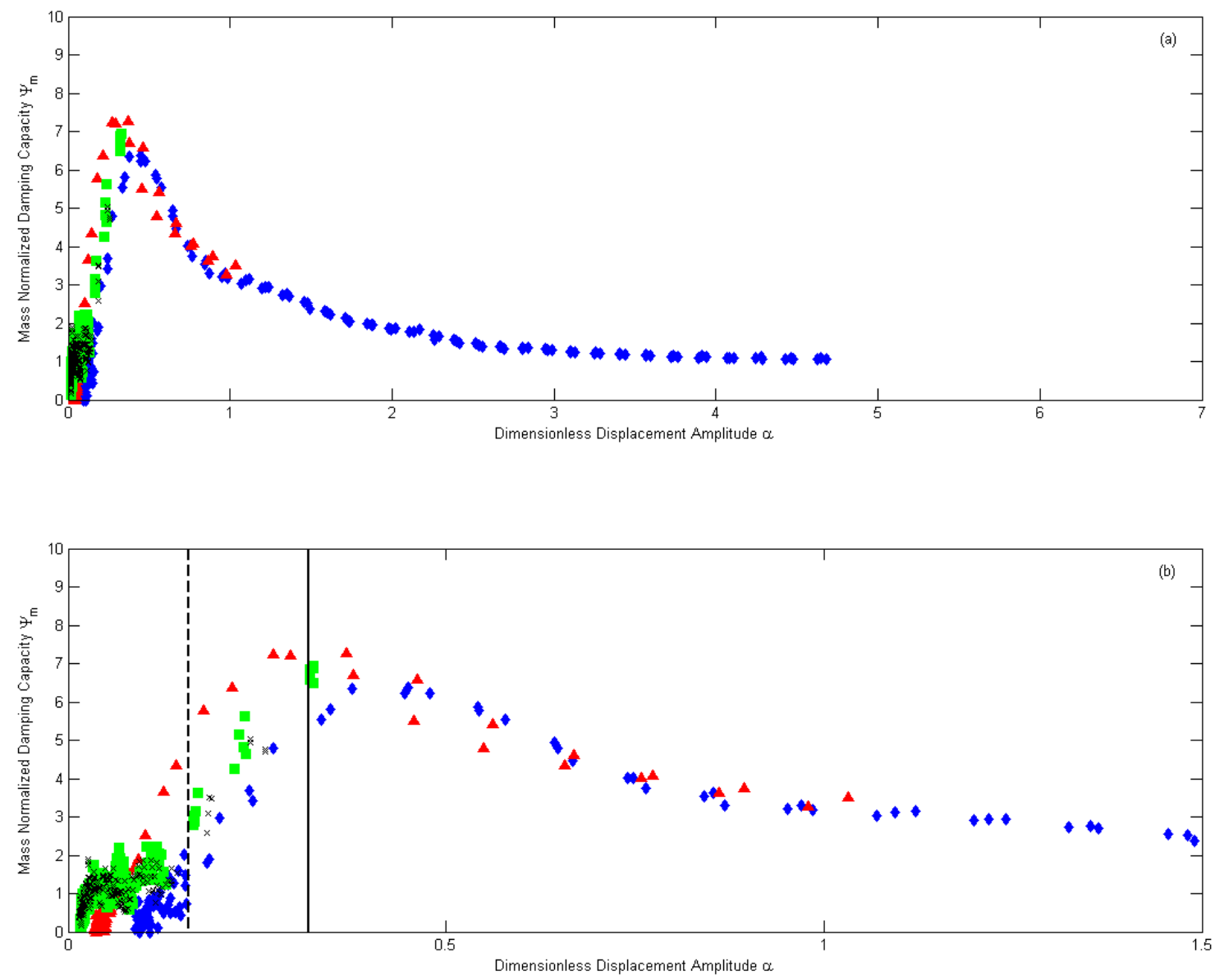

Fig. 4.8. Damping versus $\alpha$ for three cavities each with a single particle; effects of varying clearance $d$. (a) Full range of $\alpha$ tested. (b) Detail zoom showing $\alpha=1 / \pi$ (solid line) and $\alpha=1 /(2 \pi)$ (dashed line). $d_{1}=1.22 \mathrm{~mm}, \Delta d_{2}=4.95 \mathrm{~mm}, \boldsymbol{\nabla} d_{3}=14.88 \mathrm{~mm}, \mathbf{x}$ $d_{4}=19.74 \mathrm{~mm}$. For each series $U_{o}=6.0 \mathrm{~mm}$. 


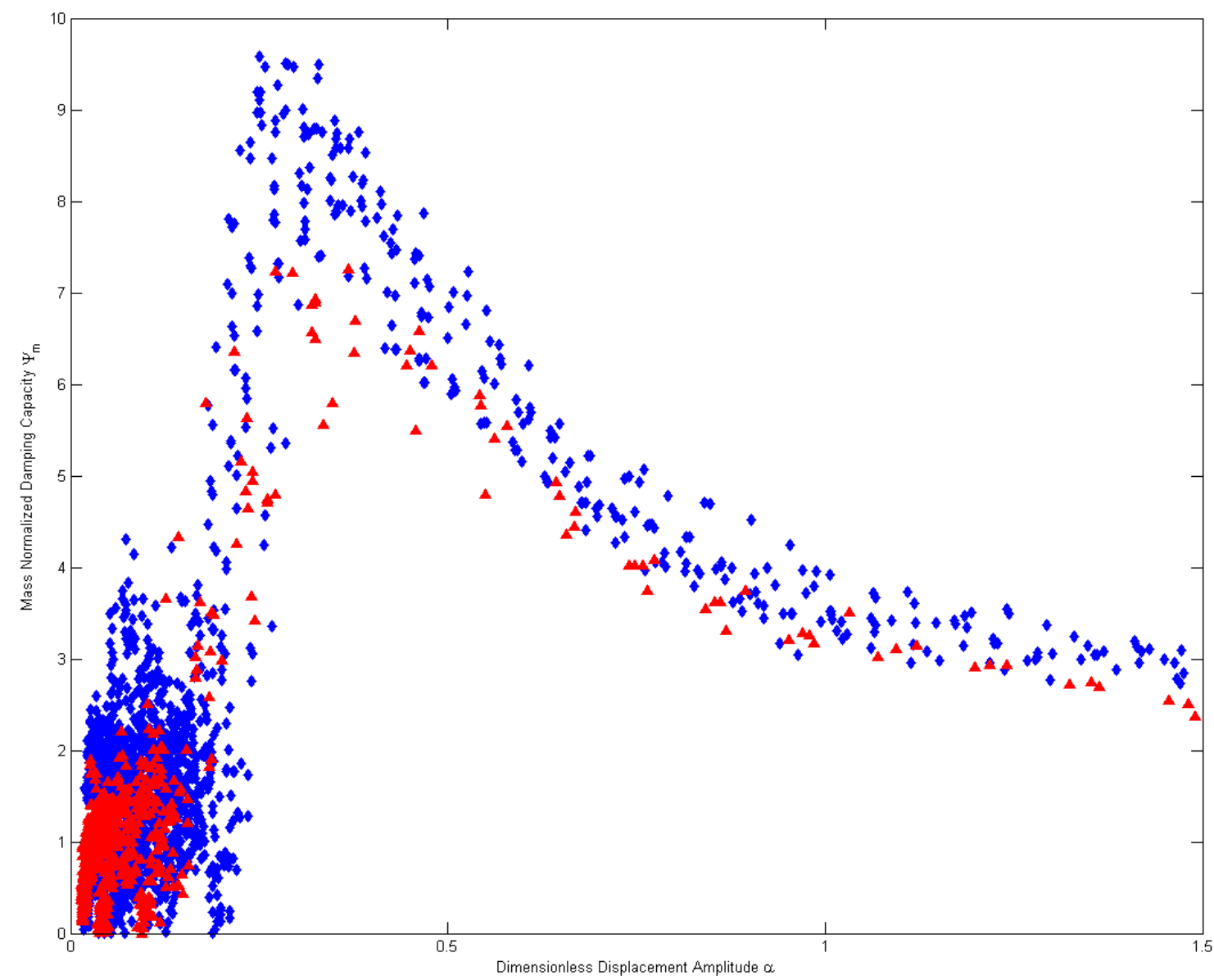

Fig. 4.9. Comparison of damping for two different particle configurations near their peak damping point. Configuration 1: a single chamber containing a single particle, $\Delta$ Configuration 2: three identical chambers each containing a single particle. 


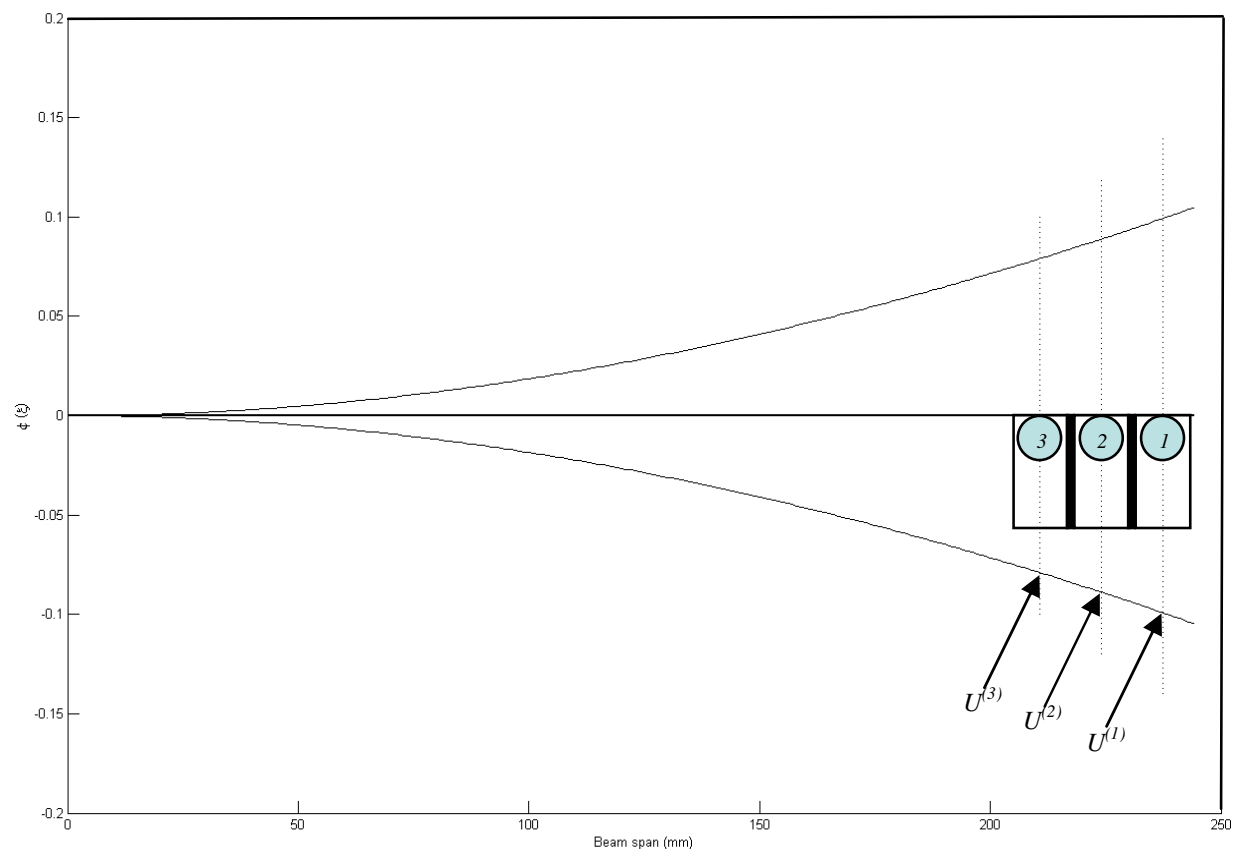

Fig. 4.10. Fundamental mode shape and placement of particles along the beam for Configuration 2 (drawn to scale). 
$>U_{o}{ }^{(2)} \omega>U_{o}{ }^{(3)} \omega$. Under these conditions, particle (1) will impact the enclosure first dissipating some amount of kinetic energy. Butt and Akl [2] have shown the impact of a damper will instantaneously change the velocity at all points in a structure. This reduces the relative velocity between the two successive particles and the enclosure. From Eq. (2.24), we conclude the resulting damping due to the impact of the second and third particles will be less than the first, $\Psi^{(3)}<\Psi^{(2)}<\Psi^{(1)}$.

These findings are of particular importance for the practical development and application of PID. Regarding design efforts for damper enclosures with individual particles in separate chambers it is recommended the cavities be arranged such that particle motion will be as uniform as possible. For example, the damper used in Configuration 2 would exhibit higher damping if the cavities had been aligned normal to the plane of vibration rather than along the span of the beam. In this orientation the particles would be expected to travel in unison as the effects of the beam's mode shape are removed.

\subsection{Configuration 3: Three Cavities Each Containing Two Particles}

The third tested damper configuration comprises three individual chambers each containing two particles. A schematic of this arrangement is given in Fig. 4.11. These particles are identical to those used in the single- particle (Section 4.1) and three-particle configurations(Section 4.2): $\quad D=9.50 \mathrm{~mm}$ and an individual mass of $3.52 \mathrm{~g}$ making the total particle mass $m_{p}=21.12$ grams. Each of the three cavities has a fixed height $h_{e}=$ $9.90 \mathrm{~mm}$ and a fixed width $w_{e}=12.19 \mathrm{~mm}$. As with the previous two configurations 


\section{Top View}

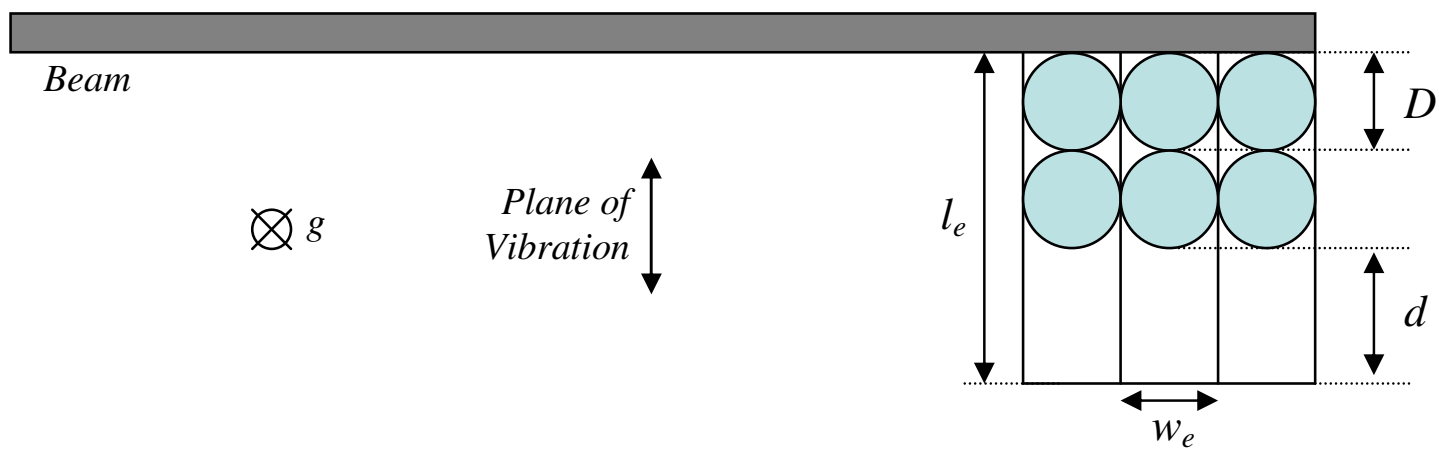

\section{Front View}

\section{Beam}

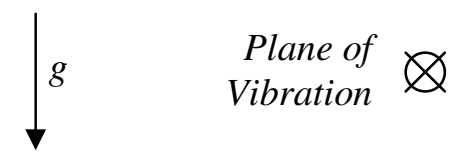

Fig. 4.11. Configuration 3: three separate cavities each containing two particles (not to scale). $N=6, D=9.50 \mathrm{~mm}, m_{p}=21.12$ grams. 
resin inserts are placed in the main PID enclosure to create the three separate cavities (see Figs. 4.2 and 4.3).

Data was collected for $d=[1.22,4.95] \mathrm{mm}$, and $U_{o}=[2.0,4.0,6.0,8.0] \mathrm{mm}$, and is presented in Fig. 4.12. It is reassuring to note that the data collapses into a single curve. A zoom view of the data is shown in Fig. 4.12b. We note that the peak does not coincide with $\alpha=1 / \pi$ nor does $\Psi_{m}$ approach zero at $\alpha=1 /(2 \pi)$. It is not surprising that the elementary single-particle model is inadequate for predicting the behavior of this configuration of six particles in three chambers. A comparison of the data obtained with the first three configurations is presented in Fig. 4.13. It is seen that $\Psi_{m}$ decreases with the number of particles.

The design implication of Fig. 4.13 is that if one can use a single particle then one achieves highest damping per unit mass. However, if one must use several particles then there is a trade off between the number of particles and the damping per unit mass.

\subsection{Configuration 4: Single Horizontal Layer of Particles}

The fourth damper configuration is a single horizontal layer of particles within one cavity. The number of particles in the layer is determined by how many "rows" of particles are used. For the enclosure used in this set of experiments a row is defined as nine particles which just span the width of the cavity when lined up (see Fig. 4.14). In this manner the clearance $d$ between the particles and enclosure is readily quantified. 

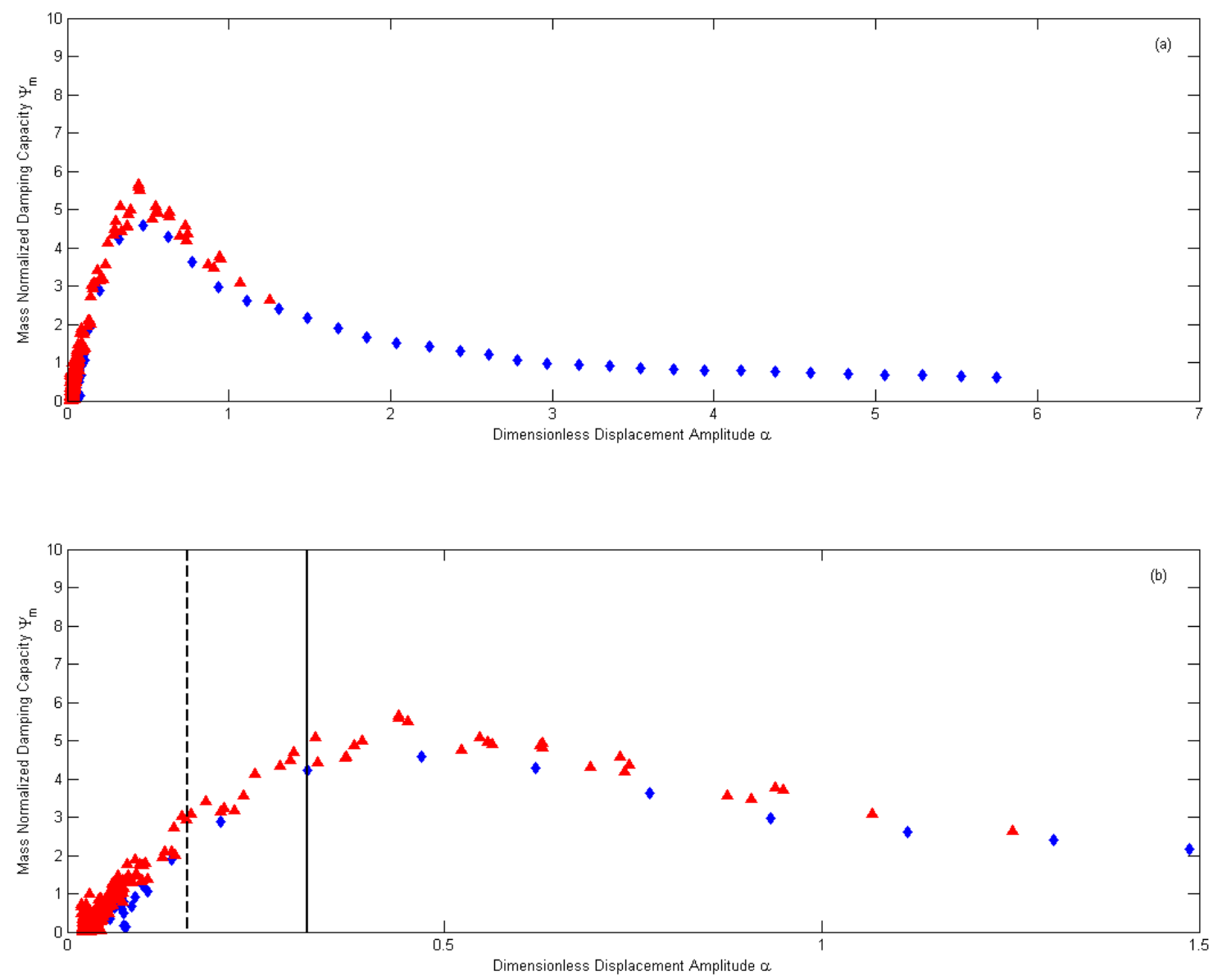

Fig. 4.12. $\Psi_{m}$ versus $\alpha$ for three cavities each containing two particles; effects of varying clearance $d$. (a) Full range of $\alpha$ tested (b) zoom showing $\alpha=1 / \pi$ (solid line) and $\alpha=$ $1 /(2 \pi)$ (dashed line). $d_{1}=1.22 \mathrm{~mm}, \Delta d_{2}=4.95 \mathrm{~mm}$. Each series contains $U_{o}=[2.0$, $4.0,6.0,8.0] \mathrm{mm}$. 


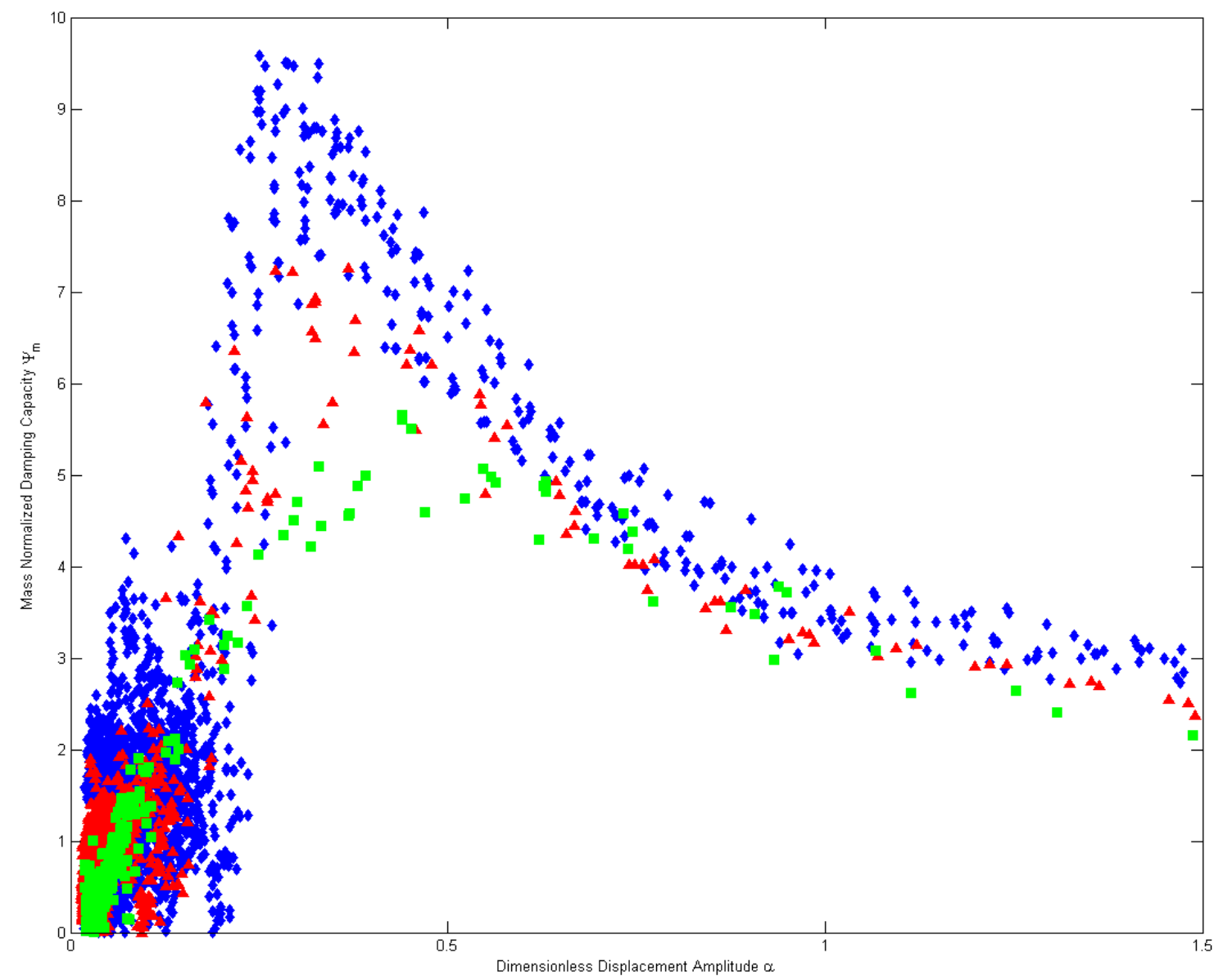

Fig. 4.13. Comparison of $\Psi_{m}$ for three different particle configurations near their peak damping points. Configuration 1: a single chamber containing a single particle, $\Delta$ Configuration 2: three identical chambers each containing a single particle, Configuration 3: three identical chambers each containing two particles. 


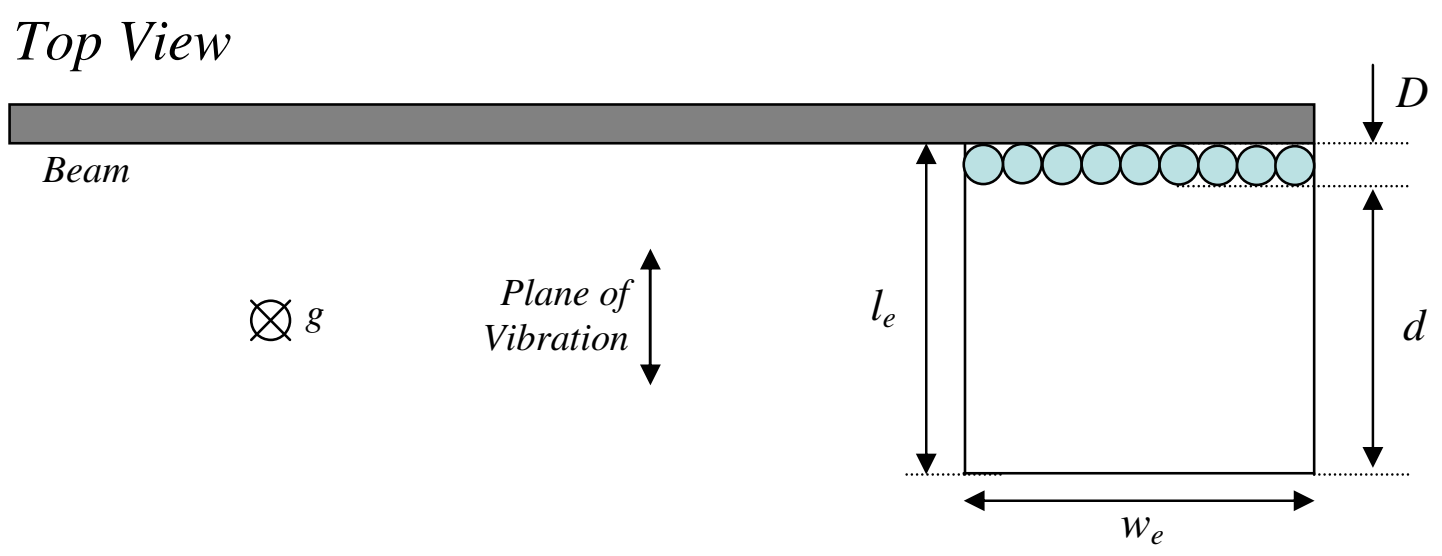

Front View

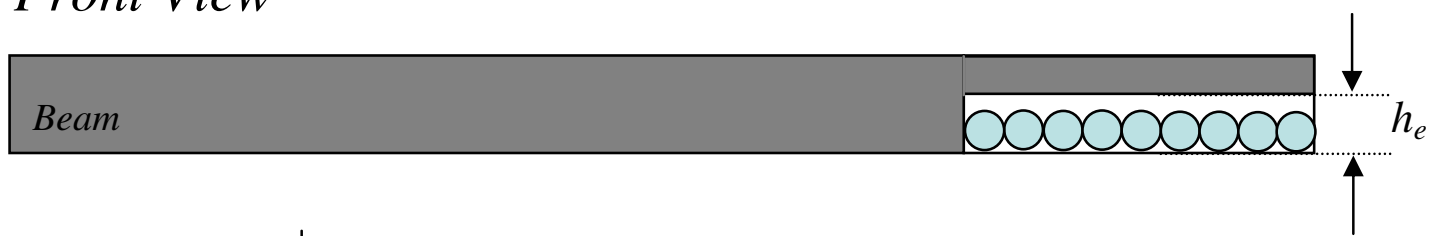

$\downarrow \quad \begin{array}{r}\text { Plane of } \\ \text { Vibration }\end{array}$

Fig. 4.14. Configuration 4: single horizontal layer with one row of particles (not to scale). $N=9, D=4.36 \mathrm{~mm}, m_{p}=3.13$ grams. 
Unlike the previous three configurations the particles are not constrained by channel-like cavities. Fig. 4.14 is a schematic of a single horizontal layer $(n=1)$ containing one row of particles, i.e. $N=9$. All particles used in these tests have a diameter $D=4.36 \mathrm{~mm}$. The enclosure has a fixed width of $w_{e}=39.8 \mathrm{~mm}$. The length of the enclosure $l_{e}$ is variable which allows setting particular values of $d$ regardless of the number of particle rows present. The height of the enclosure $h_{e}=5.00$ is set such that the particle layer is constrained to remain on the enclosure floor. This prevents particles from climbing over each other creating more that one layer of particles. Tests are performed incrementally from one row to four rows giving a particle count range of $N=9$ to 36 . A representative schematic of a single horizontal layer of particles with four rows is presented in Fig. 4.15. For each number of rows, experiments are performed for about 25 combinations of clearances $d=[1.22,2.25,4.00,4.36,4.95,7.00,10.00,14.88,19.74] \mathrm{mm}$ and initial displacements $U_{o}=[1.0,2.0,4.0,6.0,8.0,10.0] \mathrm{mm}$. The results of these tests are shown in Fig. 4.16. In the top graph (a) data is presented for $4.00<d<19.74 \mathrm{~mm}$. Attention is first drawn to data for one row ( $\diamond)$. As previously observed, for different combinations of $U$ and $d$, damping depends uniquely on $\alpha$ within the experimental scatter. The same is true for two, three and four rows. As observed in Fig. 4.13 as the number of particles increases $\Psi_{m}$ decreases and the peak damping shifts to the right. Data for the two smaller values of $d$ (less than the diameter of the particles, $D$ ) is plotted in Fig. 4.16b. For one row the data is similar to that in Fig. 4.16a. However, for two, three or four rows the peak $\Psi_{m}$ decreases rapidly. Moreover, $\Psi_{m}$ is no longer uniquely determined by $\alpha$. This is further illustrated in Fig. 4.16c where only the two row data from Fig. 4.16b is 


\section{Top View}

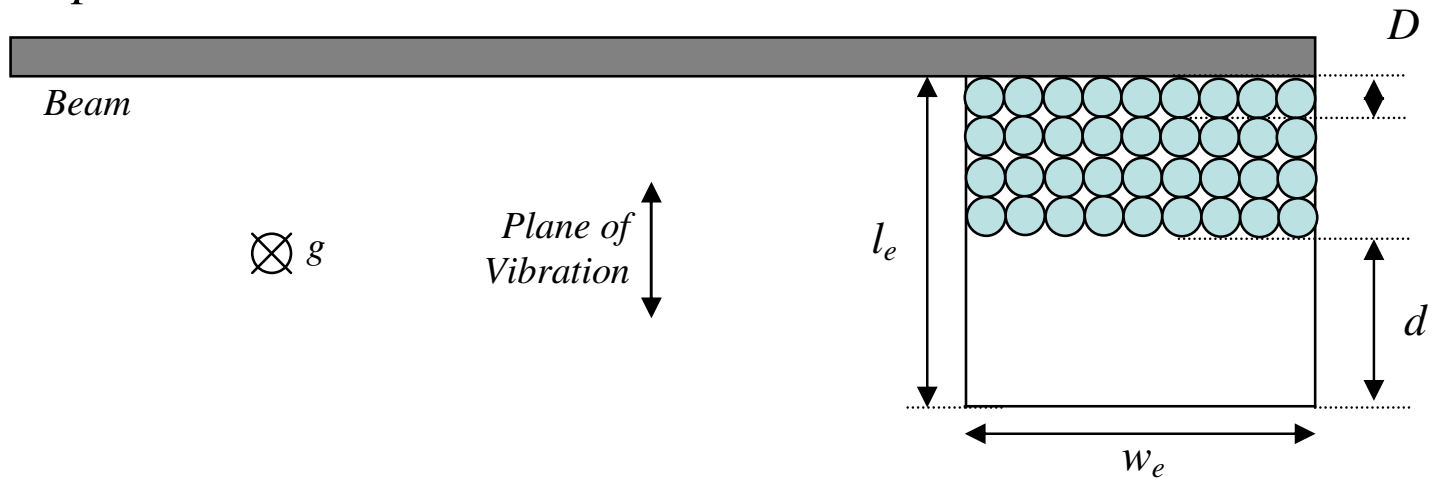

\section{Front View}
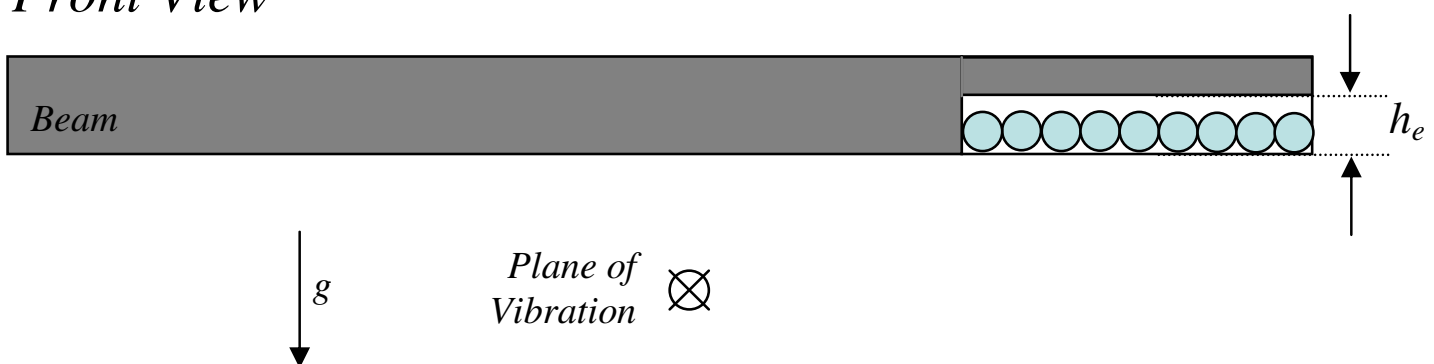

Fig. 4.15. Configuration 4: single horizontal layer with four rows of particles (not to scale). $N=36, D=4.36 \mathrm{~mm}, m_{p}=12.43$ grams. 

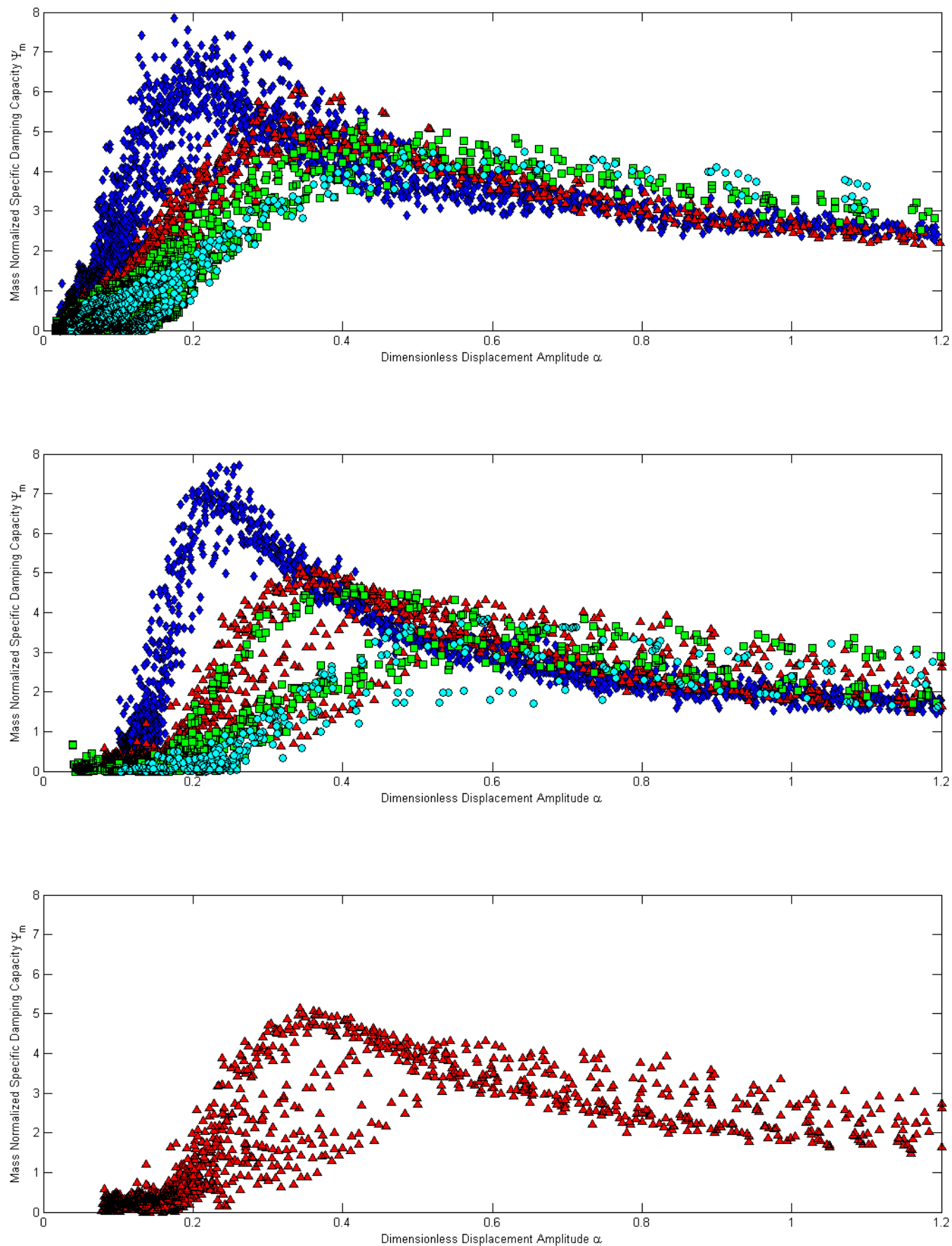

Fig. 4.16. Single horizontal layer of particles, effects of varying the number of particle rows. (a) $d=[4.00,4.36,4.95,7.00,10.00,14.88,19.74] \mathrm{mm}$. (b) $d=[1.22,2.25] \mathrm{mm}$. (c) Only two rows of particles with $d=[1.22,2.25] \mathrm{mm}$. Each series contains data with initial displacement amplitudes of $U_{o}=[1.0,2.0,4.0,6.0,8.0,10.0] \mathrm{mm}: \downarrow 1$ row, $\mathrm{N}=$ 9; $\Delta$ rows, $\mathrm{N}=18 ;-3$ rows, $\mathrm{N}=27 ; \circ 4$ rows, $\mathrm{N}=36$. 
plotted for clarity. For example note that $\alpha=0.3$ results in three different values of $\Psi_{m}$. Therefore, for practical design considerations it is advisable to use $d \geq D$ to help ensure repeatable damping performance.

\subsection{Configuration 5: Multiple Layers of Particles}

The fifth and final damper configuration comprises multiple layers of particles within a single cavity. A representative schematic of this type of configuration is shown in Fig. 4.17. Particles used for these tests are lead spheres with diameter $D=1.27 \mathrm{~mm}$. The enclosure cavity has a fixed width $w_{e}=24.38 \mathrm{~mm}$ and fixed height $h_{e}=35.31 \mathrm{~mm}$. The length of the enclosure $l_{e}$ is variable. The height of the particle bed, $h_{p}$, is varied either by adjusting $l_{e}$ and leaving the number of particles fixed or by changing the number of particles while $l_{e}$ remains constant (all results are mass normalized). Tests are conducted using three values of $l_{e}=[19.63,24.03,34.77] \mathrm{mm}$. For each enclosure length the number of particles is adjusted to achieve one of four particle bed depths, $h_{p}=[4.41$, 7.98, 11.84, 16.89] mm. For each of these twelve settings data is collected at three initial displacement values $U_{o}=[1.0,2.5,5.0] \mathrm{mm}$. Overall there are thirty six different tests each of which is repeated a minimum of three times to check data repeatability. Table 4.2 contains the test matrix performed for Configuration 5. The total number of particles $N$ is estimated based on the mass of the particle bed $m_{p}$. Supported by visual observations, the particle stacking arrangement is assumed to be a hexagonal closed pack structure as shown in Fig. 4.18 which leads to the following relation for the number of layers $n$ : 
Top View

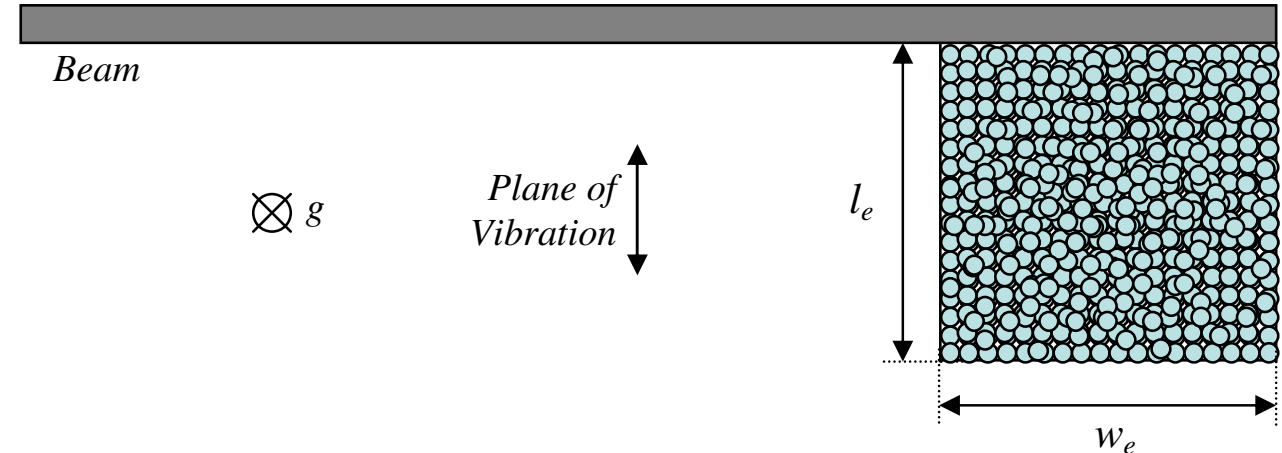

\section{Front View}

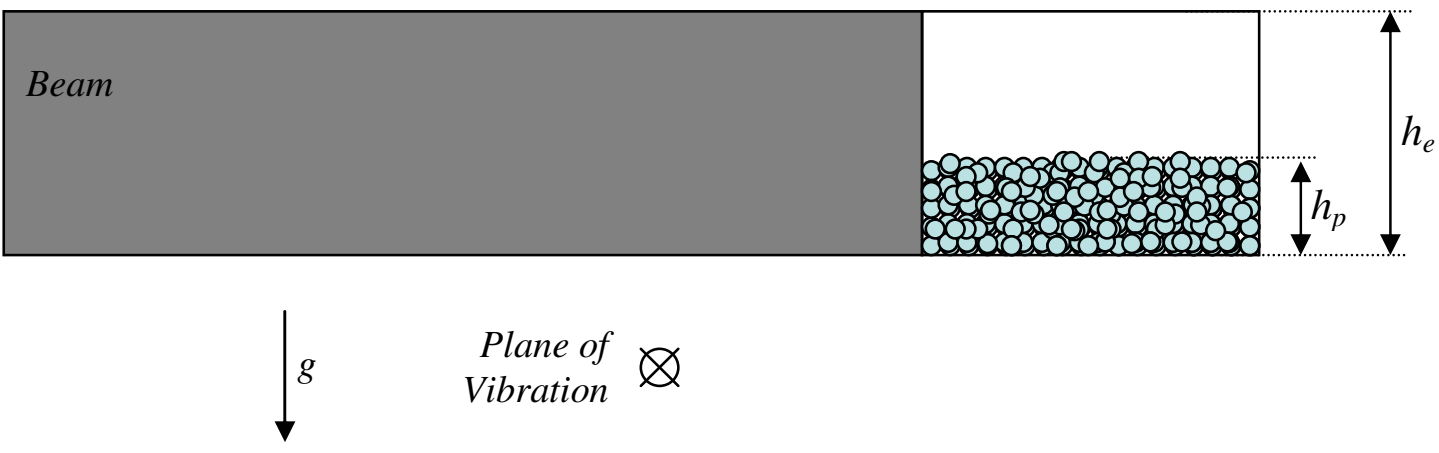

Fig. 4.17. Configuration 5: multiple layers of particles (not to scale). 
Table 4.2. Test matrix for experiments performed with multiple layers of particles in a single cavity (Configuration 5).

\begin{tabular}{|c|c|c|c|c|c|c|}
\hline Run No. & $m_{p}$ & $I_{e}$ & $\overline{h_{p}}$ & $U_{0}$ & $\bar{N}$ & $n$ \\
\hline 1 & 23.48 & 34.77 & $\overline{4.41}$ & 1.0 & 1925 & 4 \\
\hline 2 & 23.48 & 34.77 & 4.41 & 2.5 & 1925 & 4 \\
\hline 3 & 23.48 & 34.77 & 4.41 & 5.0 & 1925 & 4 \\
\hline 4 & 47.81 & 34.77 & 7.98 & 1.0 & 3919 & 7 \\
\hline 5 & 47.81 & 34.77 & 7.98 & 2.5 & 3919 & 7 \\
\hline 6 & 47.81 & 34.77 & 7.98 & 5.0 & 3919 & 7 \\
\hline 7 & 72.59 & 34.77 & 11.84 & 1.0 & 5950 & 11 \\
\hline 8 & 72.59 & 34.77 & 11.84 & 2.5 & 5950 & 11 \\
\hline 9 & 72.59 & 34.77 & 11.84 & 5.0 & 5950 & 11 \\
\hline 10 & 95.69 & 34.77 & 16.89 & 1.0 & 7843 & 15 \\
\hline 11 & 95.69 & 34.77 & 16.89 & 2.5 & 7843 & 15 \\
\hline 12 & 95.69 & 34.77 & 16.89 & 5.0 & 7843 & 15 \\
\hline$\overline{13}$ & 15.16 & 24.03 & 4.41 & 1.0 & 1243 & 4 \\
\hline 14 & 15.16 & 24.03 & 4.41 & 2.5 & 1243 & 4 \\
\hline 15 & 15.16 & 24.03 & 4.41 & 5.0 & 1243 & 4 \\
\hline 16 & 31.86 & 24.03 & 7.98 & 1.0 & 2611 & 7 \\
\hline 17 & 31.86 & 24.03 & 7.98 & 2.5 & 2611 & 7 \\
\hline 18 & 31.86 & 24.03 & 7.98 & 5.0 & 2611 & 7 \\
\hline 19 & 48.18 & 24.03 & 11.84 & 1.0 & 3949 & 11 \\
\hline 20 & 48.18 & 24.03 & 11.84 & 2.5 & 3949 & 11 \\
\hline 21 & 48.18 & 24.03 & 11.84 & 5.0 & 3949 & 11 \\
\hline 22 & 67.22 & 24.03 & 16.89 & 1.0 & 5510 & 15 \\
\hline 23 & 67.22 & 24.03 & 16.89 & 2.5 & 5510 & 15 \\
\hline 24 & 67.22 & 24.03 & 16.89 & 5.0 & 5510 & 15 \\
\hline 25 & 12.56 & 19.63 & 4.41 & $\overline{1.0}$ & 1030 & 4 \\
\hline 26 & 12.56 & 19.63 & 4.41 & 2.5 & 1030 & 4 \\
\hline 27 & 12.56 & 19.63 & 4.41 & 5.0 & 1030 & 4 \\
\hline 28 & 24.39 & 19.63 & 7.98 & 1.0 & 1999 & 7 \\
\hline 29 & 24.39 & 19.63 & 7.98 & 2.5 & 1999 & 7 \\
\hline 30 & 24.39 & 19.63 & 7.98 & 5.0 & 1999 & 7 \\
\hline 31 & 37.23 & 19.63 & 11.84 & 1.0 & 3052 & 11 \\
\hline 32 & 37.23 & 19.63 & 11.84 & 2.5 & 3052 & 11 \\
\hline 33 & 37.23 & 19.63 & 11.84 & 5.0 & 3052 & 11 \\
\hline 34 & 49.17 & 19.63 & 16.89 & 1.0 & 4030 & 15 \\
\hline 35 & 49.17 & 19.63 & 16.89 & 2.5 & 4030 & 15 \\
\hline 36 & 49.17 & 19.63 & 16.89 & 5.0 & 4030 & 15 \\
\hline
\end{tabular}



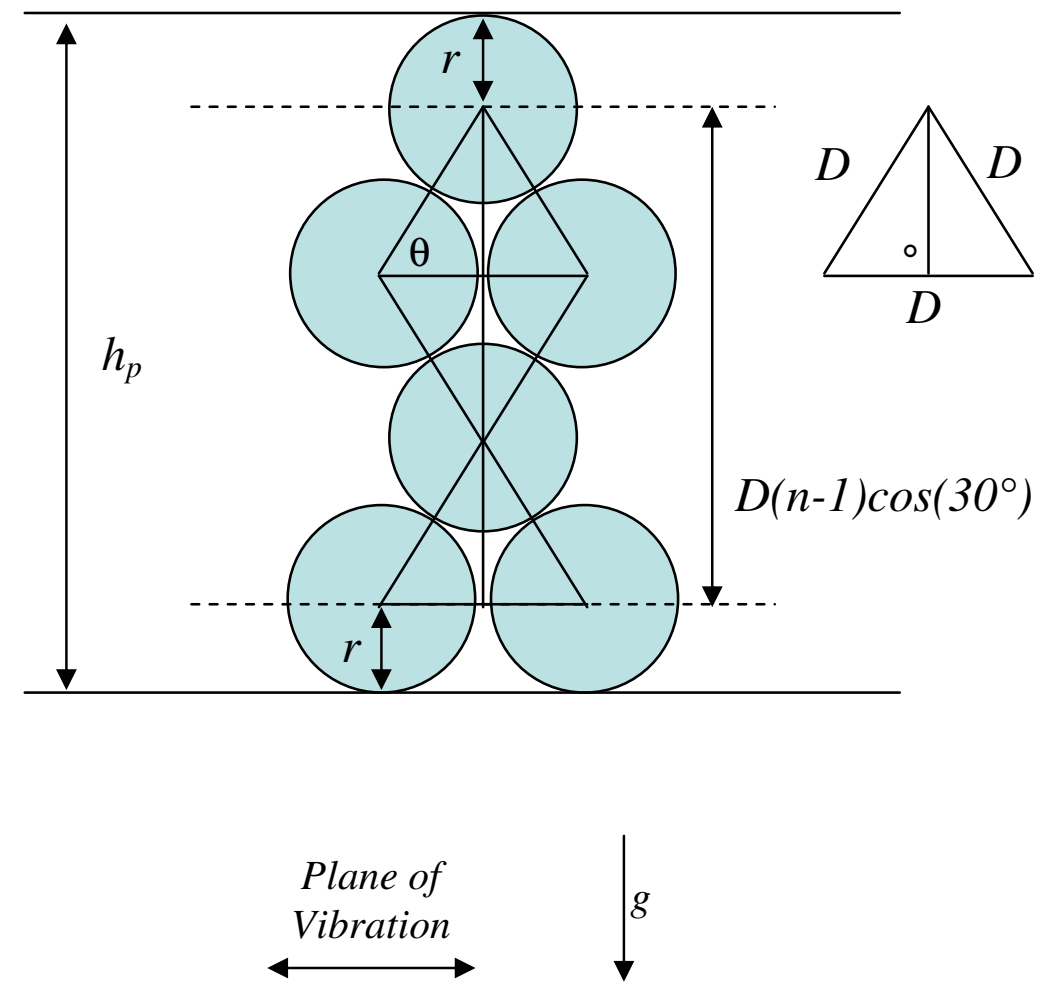

Fig. 4.18. Particle arrangement assumed for calculating the number of layers $n$ for dampers configured with multiple layers of particles (Configuration 5). 


$$
n=\frac{h_{p}-D}{D \cos \left(30^{\circ}\right)}+1
$$

In the four previous configurations the clearance parameter in the plane of vibration, $d$, was used as the normalization term in the dimensionless displacement amplitude, $\alpha=$ U/d. However, for the current configuration in which particles completely cover the bottom of the enclosure, the clearance parameter $d$ has no clear meaning and thus a different dimensionless parameter is sought. It will be shown that the dimensionless parameter $\beta$ which is a function of the enclosure acceleration, gravity, particle bed depth, enclosure height, and particle diameter serves this purpose:

$$
\beta=f\left(U \omega^{2}, g, h_{p}, h_{e}, D\right)
$$

Consider two identical enclosures, one with only a few layers of particles ( $\operatorname{small} h_{p}$ ) and the second with a substantially greater number of layers (large $h_{p}$ ). For both cases assume $h_{e} \gg h_{p}$ so the particles do not interact with the enclosure ceiling. The acceleration required to excite the top layer (and subsequent underlying layers) is the same for both cases. Thus, for a given acceleration amplitude the approximate total number of particles in motion is the same for both cases. However, the percentage of particles moving in the first enclosure (with small $h_{p}$ ) will be larger than that of the second enclosure (with large $h_{p}$ ). Therefore in the second case greater acceleration is required to excite the same percentage of particles and achieve the same magnitude of $\Psi_{m}$. This is supported by Fig. 4.19 which contains the data collected with $l_{e}=34.77 \mathrm{~mm}$ 
(Runs 1-12, Table 4.2) plotted as a function of the dimensionless acceleration amplitude $\Gamma=U \omega^{2} / g$. As expected, for sufficiently small $\Gamma$, such that only a fraction of the particles are excited, the damping decreases with the total number of particles. Moreover, the acceleration amplitude at which peak damping is achieved increases with $h_{p}$

It should also be noted from Fig. 4.19 that for each value of $h_{p}$ the damping falls into a single curve for all $U_{o}$ tested. This behavior has been observed in all previous configurations with the exception of Configuration 4 when the clearance $d<D$. Also, the peak damping $\Psi_{m} \approx 3.25$ is appreciably smaller than that in all previous configurations. Recall from Fig. 4.13 that peak damping for Configuration 1 was $\Psi_{m} \approx$ 9.5. This reduction in peak mass normalized damping is expected based on the trend from previous configurations where increasing the number of particles leads to a decrease in maximum $\Psi_{m}$. The reduction in peak damping due to increasing the number of particles can even be observed within Fig. 4.19 as more particles are added to increase $h_{p}$

As an extension of the data presented in Fig. 4.19, one can imagine the effects of increasing $h_{p}$ such that the enclosure fill ratio $H=h_{p} / h_{e} \rightarrow 1$. Clearly, as the fill ratio approaches unity the particle bed becomes compacted and particle motion will become increasingly restricted. It is then expected that as the height ratio increases damping will approach zero. Similar observations have been made in the past by Hollkamp and 


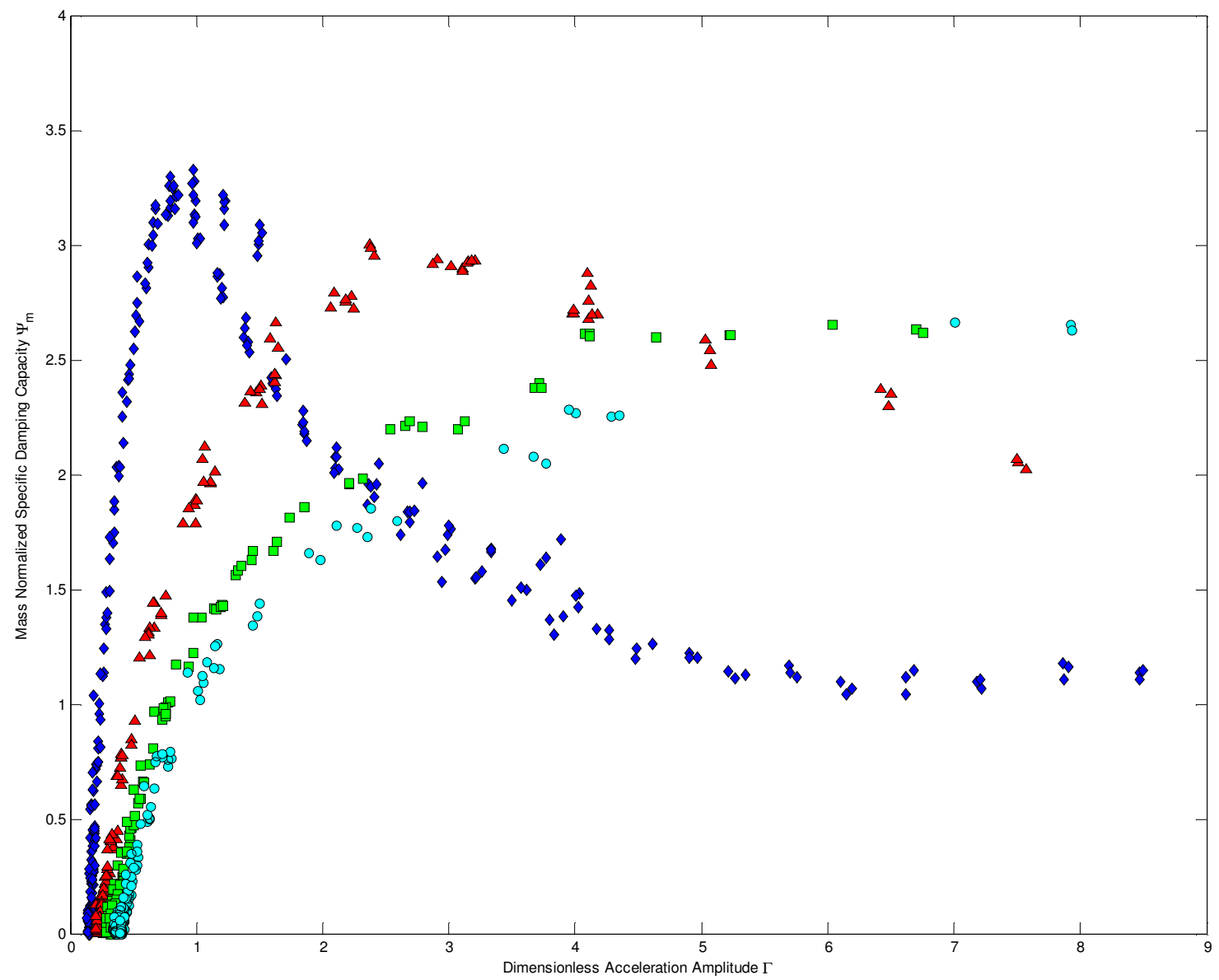

Fig. 4.19. Damping versus $\Gamma$ for multiple layers of particles; effects of changing $h_{p}$ by adjusting $m_{p}$. Constant $l_{e}=34.77 \mathrm{~mm}, U_{o}=[1.0,2.5,5.0] \mathrm{mm}$. $h_{p}=4.41 \mathrm{~mm}, \Delta h_{p}=$ $7.98 \mathrm{~mm}, \square h_{p}=11.84 \mathrm{~mm}, \bullet h_{p}=16.89 \mathrm{~mm}$. 
Gordon [22].

We next consider the effect of the particle diameter. With reference to Fig. 4.20 consider two enclosures with the same particle bed depth $h_{p}$ but with different particle diameters $D$. With reference to Fig. 4.18, based on equilibrium considerations, it can be shown that for any angle $\theta$ the critical dimensionless acceleration amplitude $\Gamma=U \omega^{2} / g$ at which the uppermost particles will begin to move is

$$
\Gamma_{c r}=\frac{1}{\tan (\theta)}
$$

For the current configuration depicted in Fig. 4.20, $\theta=60^{\circ}$ irrespective of the particle diameter. As such, the top layer of particles in both cases will begin to move at the same value of $\Gamma_{c r}=1 / \sqrt{3}$. The percentage of particle mass in motion for the enclosure with large diameter particles will be greater than that of the smaller diameter particles. Therefore, it is expected $\Psi_{m}$ will be dependent on the ratio of the particle diameter to the bed depth, $\delta=D / h_{p}$.

From the discussion above it is clear damping should depend on the dimensionless terms $\Gamma, H$, and $\delta$. The expectation that $\Psi_{m}$ should increase with $\delta$ and decrease with fill ratio further suggests the following dimensionless parameter which captures the geometry of the particle configuration:

$$
\beta=\frac{\Gamma \delta}{H}=\frac{U \omega^{2} D}{g\left(\frac{h_{p}^{2}}{h_{e}}\right)}
$$


Accordingly, all the data shown in Fig. 4.19 is presented as a function of $\beta$ in Fig. 4.21. It is encouraging to note that all data collapse into a single curve.

With reference to Table 4.2, similar data for the two additional values of $l_{e}=[24.03$, 19.63] $\mathrm{mm}$ is presented as a function of $\beta$ in Figs. 4.22 and 4.23 respectively. Once again for each value of $h_{p}$ the damping falls into a single curve for all $U_{o}$ tested. These results can provide guidance in the choice of particle and enclosure geometry to target peak damping at critical acceleration levels. 
Side View
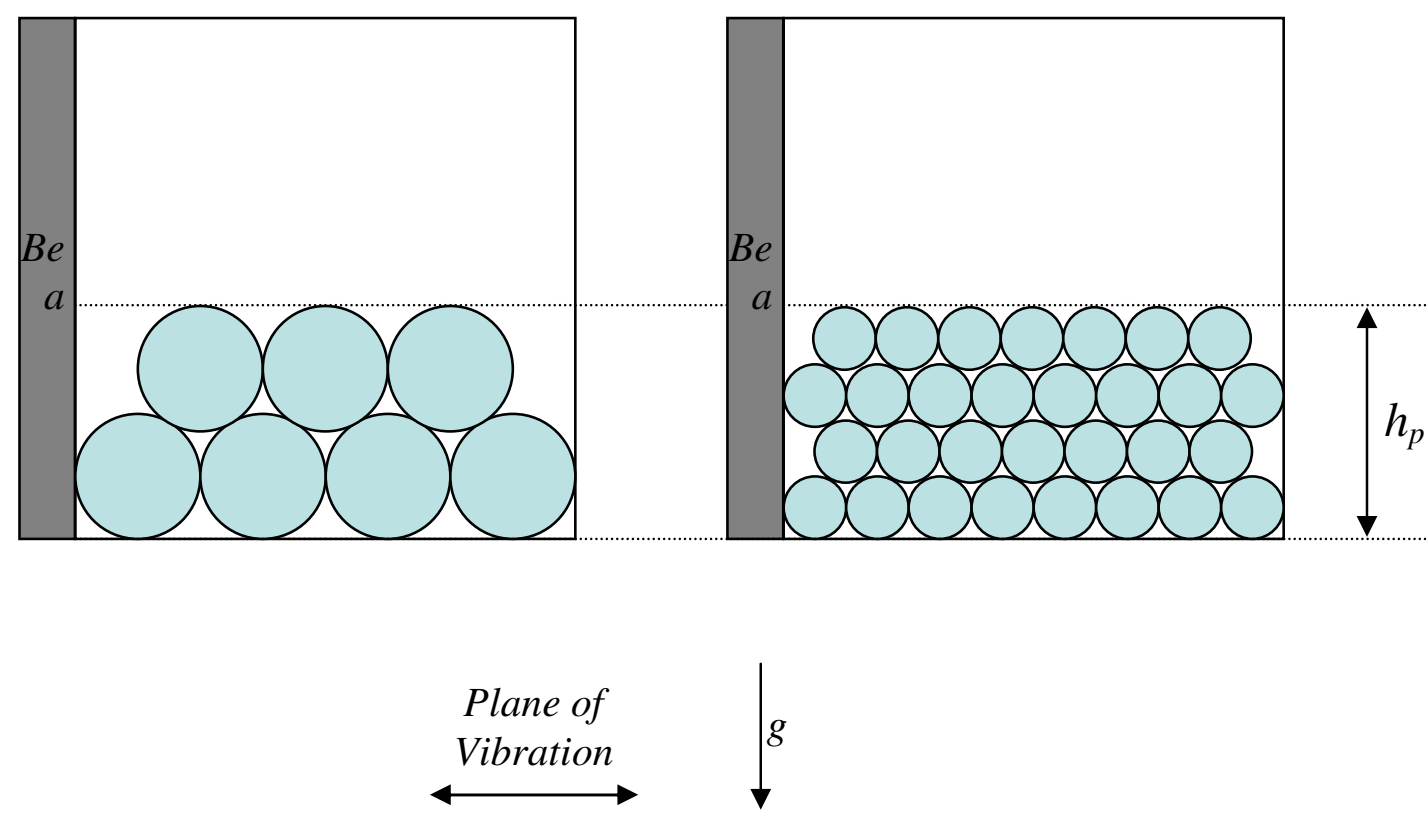

Fig. 4.20. Two enclosures with the same $h_{p}$ but different particle diameters. 


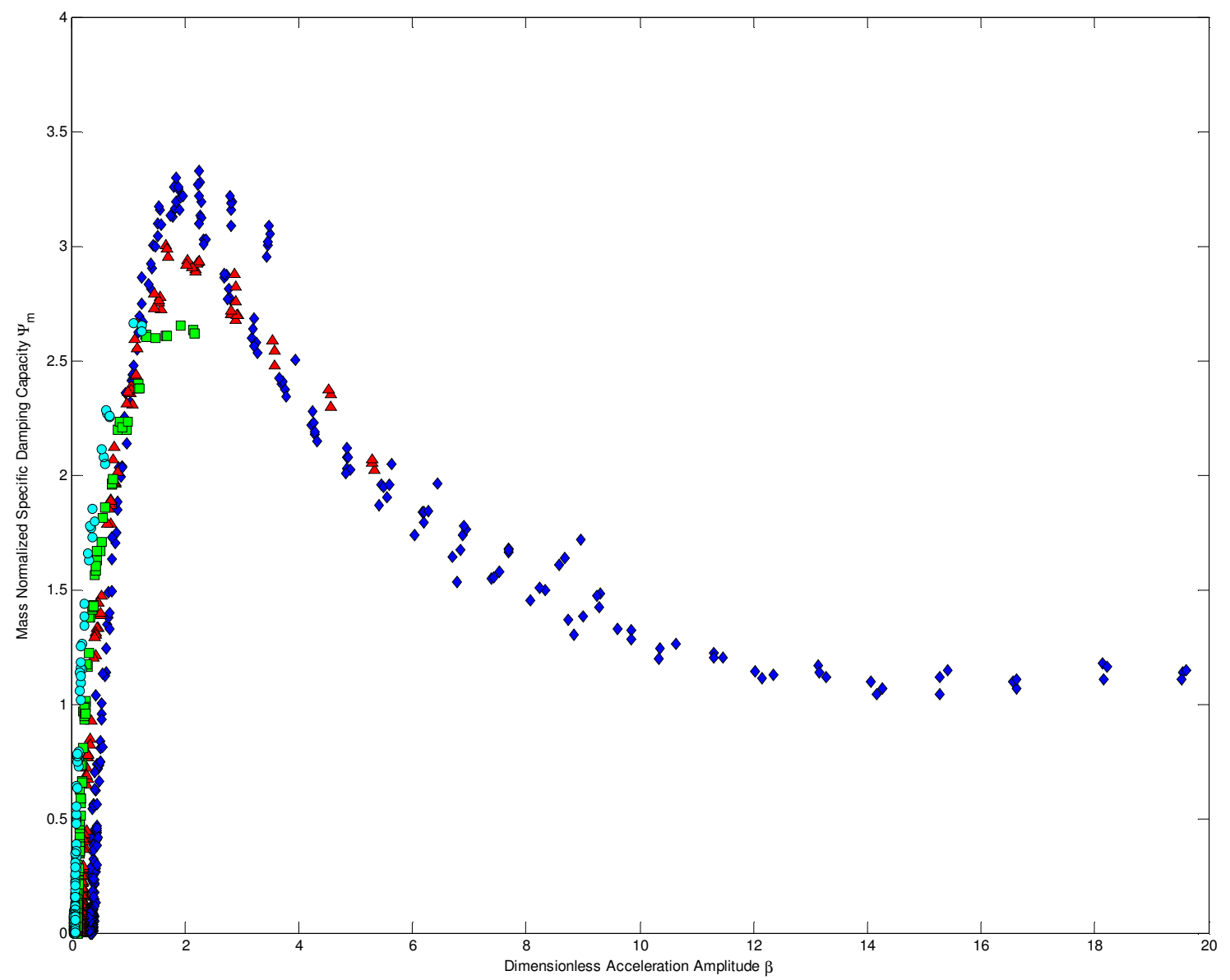

Fig. 4.21. Damping versus dimensionless acceleration amplitude $\beta$ for multiple layers of particles; effects of changing $h_{p}$ by adjusting $m_{p}$, constant $l_{e}=34.77 \mathrm{~mm}$. $U_{o}=[1.0,2.5$, $5.0] \mathrm{mm} . h_{p}=4.41 \mathrm{~mm}, \Delta h_{p}=7.98 \mathrm{~mm}, \square h_{p}=11.84 \mathrm{~mm}, \bullet h_{p}=16.89 \mathrm{~mm}$. 


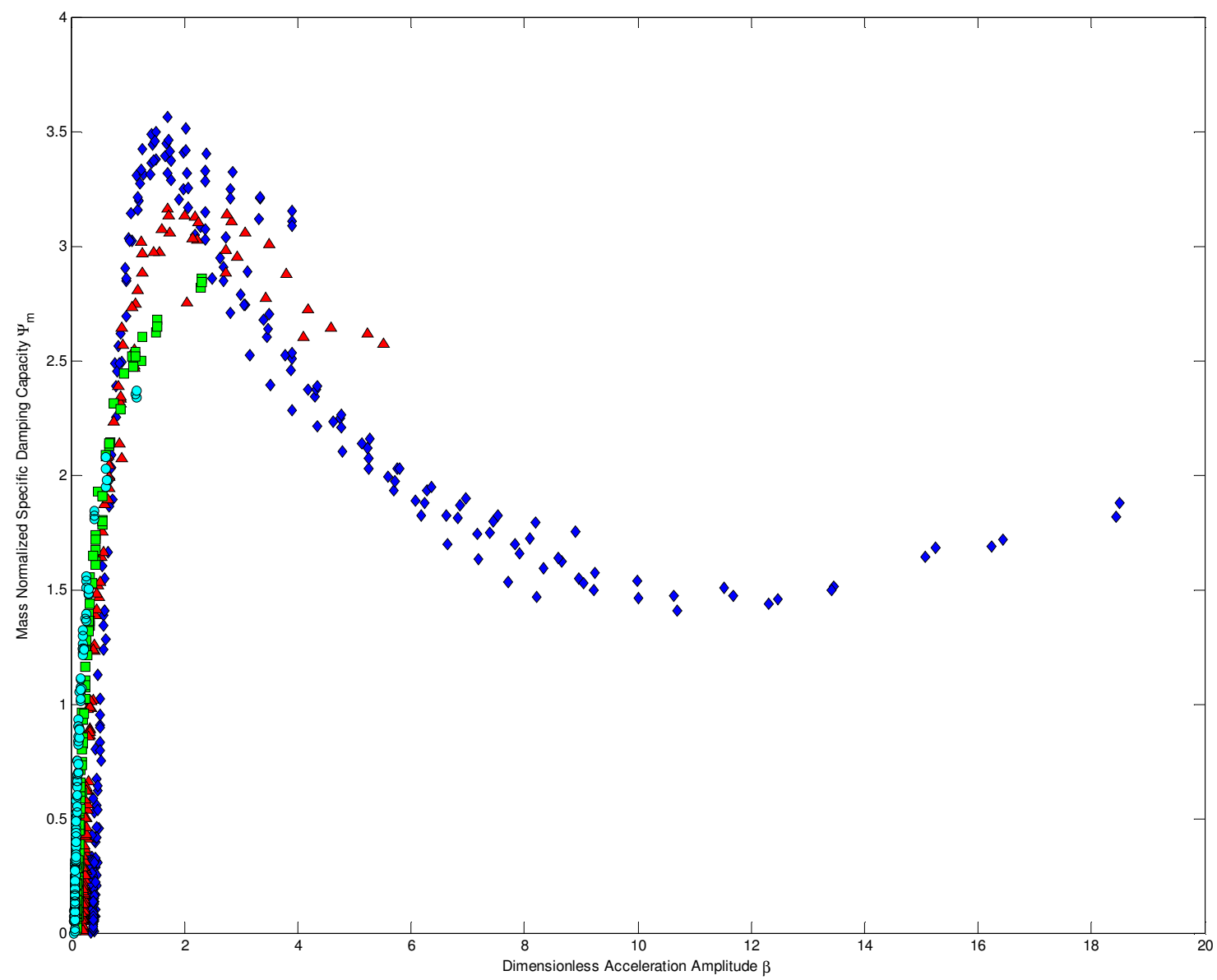

Fig. 4.22. Damping versus dimensionless acceleration amplitude $\beta$ for multiple layers of particles; effects of changing $h_{p}$ by adjusting $m_{p}$, constant $l_{e}=24.03 \mathrm{~mm}$. $U_{o}=[1.0,2.5$, $5.0] \mathrm{mm} . h_{p}=4.41 \mathrm{~mm}, \Delta h_{p}=7.98 \mathrm{~mm}, \square h_{p}=11.84 \mathrm{~mm}, \bullet h_{p}=16.89 \mathrm{~mm}$. 


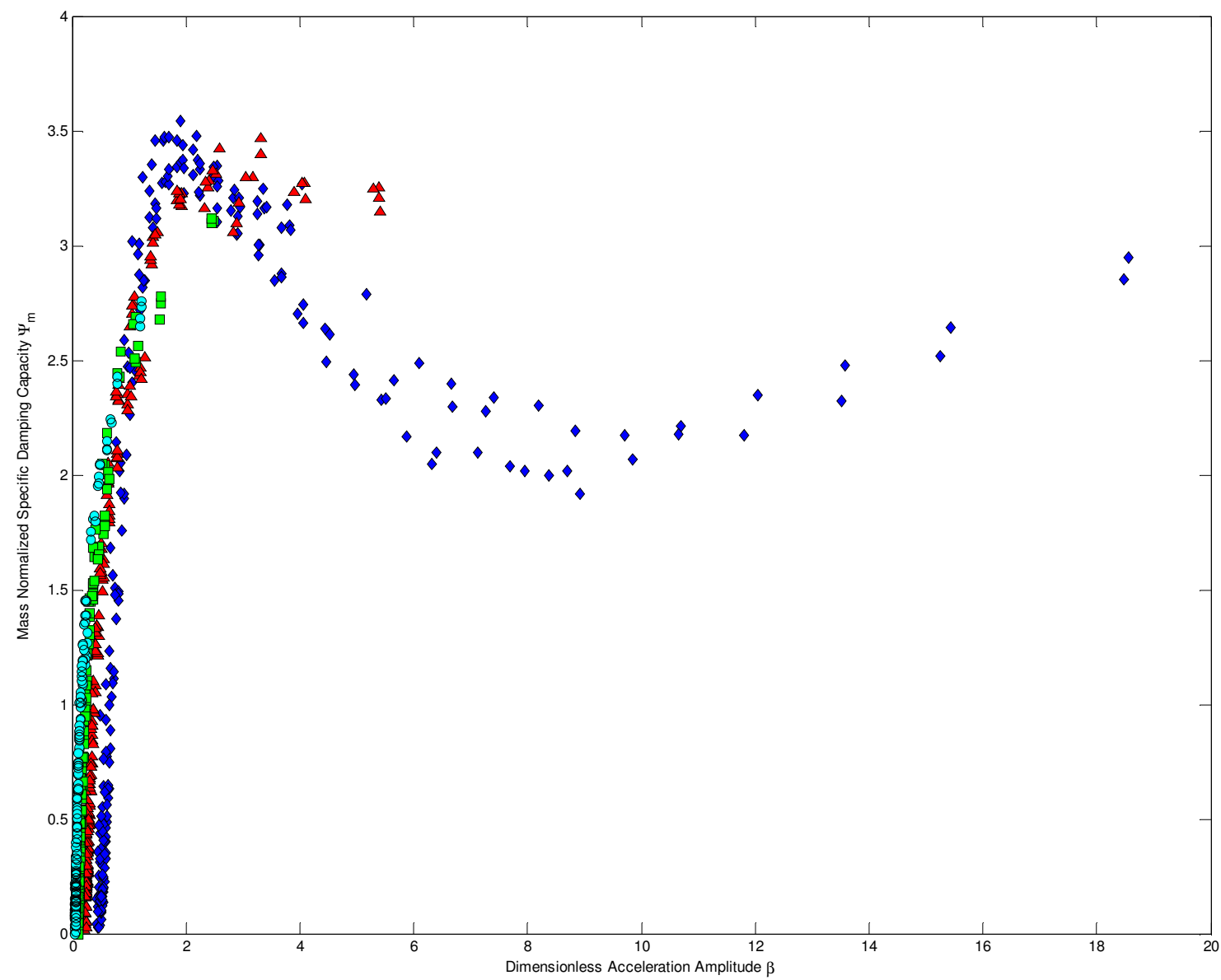

Fig. 4.23. Damping versus dimensionless acceleration amplitude $\beta$ for multiple layers of particles; effects of changing $h_{p}$ by adjusting $m_{p}$, constant $l_{e}=19.63 \mathrm{~mm}$. $U_{o}=[1.0,2.5$, $5.0] \mathrm{mm} . h_{p}=4.41 \mathrm{~mm}, \Delta h_{p}=7.98 \mathrm{~mm}, \square h_{p}=11.84 \mathrm{~mm}, \bullet h_{p}=16.89 \mathrm{~mm}$. 


\section{CONCLUSION}

The basic conditions for achieving maximum and minimum kinetic energy transfer in a PID system vibrating in the horizontal plane have been analytically evaluated. The characteristics of five distinct configurations of particle impact dampers have been experimentally investigated beginning with a single particle and progressing to multiple layers of particles.

For horizontal PID configurations with a clearly definable clearance parameter between the particle(s) and enclosure wall it has been shown $\Psi_{m}$ may be viewed as a function of a single variable $\alpha=U / d$ with the exception of a single horizontal layer of particles when $d$ $<D$. For PID configurations with multiple layers of particles for which $\alpha$ has no meaning a new dimensionless acceleration parameter $\beta$ is proposed and demonstrated. It has been shown PID devices with fewer particles deliver better damping efficiency per unit mass. However, for dampers with fewer particles the displacement range over which peak $\Psi_{m}$ is generated is very restricted. This can be overcome with the use of multiple cavities each tuned to different target displacements or acceleration magnitudes. For many practical applications to continuous systems (such as a cantilever beam) care should be exercised in the placement of multiple particle cavities. It has been demonstrated that non-uniform particle motion resulting in successive impacts significantly reduces the damping efficiency per unit mass if all cavities are tuned to the same target displacement. The analytical and experimental observations presented can 
provide guidance for the practical and efficient implementation of particle impact dampers to horizontally oscillating systems. 


\section{REFERENCES}

[1] S. Ema, E. Marui, A fundamental study on impact dampers. Int. J. Mach. Tools Manufact 34 (1994) 407-421.

[2] A.S. Butt, F.A. Akl, Numerical model of impact-damped continuous systems. Journal of Engineering Mechanics 123 (1997) 384-392.

[3] R.D. Friend and V.K. Kinra, Particle impact damping. Journal of Sound and Vibration 233 (2000) 93-118.

[4] K.S. Marhadi, V.K. Kinra, Particle impact damping: effect of mass ratio, material, and shape. Journal of Sound and Vibration 283 (2005) 433-448.

[5] M.R. Duncan, C.R. Wassgren, C.M. Krousgrill, The damping performance of a single particle impact damper. Journal of Sound and Vibration 286 (2005) 123144.

[6] C.J. Wu, W.H. Liao, M.Y. Wang, Modeling of granular particle damping using multiphase flow theory of gas-particle. Journal of Vibration and Acoustics 126 (2004) 196-201.

[7] K. Mao, M.Y. Wang, Z. Xu, T. Chen, DEM simulation of particle damping. Powder Technology 142 (2004) 154-165.

[8] S.F. Masri, General motion of impact dampers. The Journal of the Acoustical Society of America 47 (1970) 229-237.

[9] J.J. Moore, A.B. Palazzolo, R. Gadangi, T.A. Nale, S.A. Klusman, G.V. Brown, A.F. Kascak, A forced response analysis and application of impact dampers to rotordynamic vibration suppression in a cryogenic environment. Journal of Vibration and Acoustics 117 (1995) 300-310.

[10] J.M. McElhaney, A. Palazzolo, A. Kascak, Modeling and simulation methods for MDOF structures and rotating machinery with impact dampers. Journal of Engineering for Gas Turbines and Power 119 (1997) 436-445.

[11] K. Yasuda, M. Toyoda, The damping effect of an impact damper. Bulletin of the JSME 21 (1978) 424-430.

[12] C.N. Bapat, S. Sankar, Single unit impact damper in free and forced vibration. Journal of Sound and Vibration 99 (1985) 85-94. 
[13] C. Saluena, S.E. Esipov, T. Poschel, S. Simonian, Dissipative properties of granular ensembles. Proceedings of the SPIE Conference on Smart Structures and Materials: Passive Damping and Isolation, San Diego, March 1998, pp. $32-$ 42.

[14] M. Saeki, Impact damping with granular materials in a horizontally vibrating system. Journal of Sound and Vibration 251 (2002) 153-161.

[15] M. Saeki, Analytical study of multi-particle damping. Journal of Sound and Vibration 281 (2005) 1133-1144.

[16] A. Papalou, S.F. Masri, An experimental investigation of particle dampers under harmonic excitation. Journal of Vibration and Control 4 (1998) 361-379.

[17] N. Popplewell, S.E. Semercigil, Performance of the bean bag impact damper for a sinusoidal external force. Journal of Sound and Vibration 133 (1989) 193-223.

[18] C. Cempel, G. Lotz, Efficiency of vibrational energy dissipation by moving shot. Journal of Structural Engineering 119 (1993) 2642-2652.

[19] F.S. Tse, I.E. Morse, R.T. Hinkle, Mechanical Vibrations Theory and Applications, $2^{\text {nd }}$ Ed. Allyn and Bacon, Inc., Boston, 1978.

[20] W.G. Bickley, A. Talbot, An Introduction to the Theory of Vibrating Systems, Clarendon Press, Oxford, UK, 1961.

[21] I.A. Karnovsky, O.I. Lebed, Free Vibrations of Beams and Frames: Eigenvalues and Eigenfunctions, McGraw-Hill, USA, 2004.

[22] J.J. Hollkamp, R.W. Gordon, Experiments with particle damping. Proceedings of SPIE Conference on Smart Structures and Materials: Passive Damping and Isolation, San Diego, March 1998, pp. 2-12. 


\section{VITA}

Name: $\quad$ Bryan Lee Witt

Address: $\quad$ L-3 Communications Integrated Systems

7500 Maehr Road (M/S 1134)

Waco, TX 76705-1647

Email Address: bryan.l.witt@1-3com.com

Education: $\quad$ B.S., Mechanical Engineering, The University of Texas at Tyler, 2003

M.S., Aerospace Engineering, Texas A\&M University, 2011 